\title{
THE EFFECTS OF KINEMATIC CONDENSATION ON INTERNALLY RESONANT FORCED VIBRATIONS OF SHALLOW HORIZONTAL CABLES
}

\section{NARAKORN SRINIL \& GIUSEPPE REGA*}

Dipartimento di Ingegneria Strutturale e Geotecnica, Università di Roma La Sapienza, via A. Gramsci 53, Roma 00197, Italy

Number of Main-Text Pages: 26

Number of Figures: 10

Number of Tables: 4

Number of Equations: 27

*Corresponding Author.

Address: Dipartimento di Ingegneria Strutturale e Geotecnica, Università di Roma La Sapienza, via A. Gramsci 53, Roma 00197, Italy.

Telephone: +39-06-49919195

Fax: +39-06-49919192

E-mail: giuseppe.rega@uniroma1.it 


\title{
THE EFFECTS OF KINEMATIC CONDENSATION ON INTERNALLY RESONANT FORCED VIBRATIONS OF SHALLOW HORIZONTAL CABLES
}

\author{
NARAKORN SRINIL \& GIUSEPPE REGA \\ Department of Structural and Geotechnical Engineering, University of Rome 'La Sapienza', \\ via A. Gramsci 53, Rome 00197, Italy
}

\begin{abstract}
This study aims at comparing non-linear modal interactions in shallow horizontal cables with kinematically non-condensed vs condensed modeling, under simultaneous primary external and internal resonances. Planar 1:1 or 2:1 internal resonance is considered. The governing partial-differential equations of motion of non-condensed model account for spatiotemporal modification of dynamic tension, and explicitly capture non-linear coupling of longitudinal/vertical displacements. On the contrary, in the condensed model, a single integrodifferential equation is obtained by eliminating the longitudinal inertia according to a quasi-static cable stretching assumption, which entails spatially-uniform dynamic tension. This model is largely considered in the literature. Based on a multi modal discretization and a second-order multiple scales solution accounting for higher-order quadratic non-linear effects of infinite modes, coupled/uncoupled dynamic responses and the associated stability are evaluated by means of frequency- and force-response diagrams. Direct numerical integrations confirm the occurrence of amplitude-steady or -modulated responses. Non-linear dynamic configurations and tensions are also examined. Depending on internal resonance condition, system elasto-geometric and control parameters, the condensed model may lead to significant quantitative and/or qualitative discrepancies, against the non-condensed model, in the evaluation of resonant dynamic responses, bifurcations and maximal/minimal stresses. Results of even shallow cables reveal meaningful drawbacks of the kinematic condensation and allow us to detect cases where the more accurate non-condensed model has to be used.
\end{abstract}

Keywords: Horizontal cable, Kinematic condensation, Modal interaction, Non-linear forced vibration, Primary resonance, Internal resonance 


\section{INTRODUCTION}

A considerable number of publications have been concerned with geometrically non-linear vibrations of elastic suspended cables. Most of them are devoted to horizontal and shallow cables, whose non-linear modal interactions due to internal resonance phenomena are quite interesting subjects. Understanding of cable structural mechanics and non-linear dynamic behaviors due to internal/external resonance effects has been obtained by various analytical, numerical and experimental treatments [1].

As regards mechanical modeling and formulation, some simplifications, worth easing the analytical procedures, are frequently found in the relevant literature. The kinematic condensation technique is a well-established one, which considers zeroing the longitudinal inertia based on the quasi-static stretching assumption of the cable in motion, and links the corresponding longitudinal displacements to the transversal ones. As far as linear vibrations are concerned, the kinematically condensed model proves to be very useful for, e.g., identifying the low-order natural frequencies and modes of low-extensible and shallow cables [2]. However, when moving towards the non-linear regime, this model essentially disregards higher-order contributions of longitudinal dynamic deformation to the physics of the problem, by admitting spatiallyindependent non-linear stretching in cable kinematics. In other words, the system longitudinal and transversal dynamics are considered as non-linearly uncoupled, and the system vibrations are governed by integro-differential equations describing the solely transversal motion. In contrast, by accounting for both spatial and temporal variability of cable stretching via exact $[3,4]$ or approximate $[5,6]$ element kinematics, overall non-linear coupling is captured through the ensuing partial-differential equations (PDEs) of motion, and the space-time varying non-linear dynamic responses and tensions can be thoroughly assessed.

From a modal interaction viewpoint, suspended cables exhibit various activable planar and non-planar (e.g., 1:1, 2:1) internal resonances due to the geometrical combination of quadratic and cubic (cable sag and stretching) nonlinearities and to the vanishing non-linear orthogonality 
properties of modes $[7,8]$. For a non-planar interaction, both 2:1 and 1:1 resonances have been investigated in [9-11] and [12-13], respectively, whereas multiple internal resonances involving several planar/non-planar modes have been explored, e.g., in [14-15]. All of these analytical studies, which describe fundamental and rich forced dynamics of a weakly non-linear system, were based on the condensed horizontal cable model. More recently, based on the noncondensed model [5], the 2:1 resonance effects on non-linear planar free vibrations have been highlighted for both horizontal/inclined cables [6]. Within an independent numerical framework, the strong modal interaction features characterizing large-amplitude free or forced vibrations due to internal resonance effects have been investigated in [3-4] and [16-17] through a finite difference- or finite element-based, non-condensed, cable model, respectively.

In this study, we aim at making a thorough comparison of cable non-linear forced responses with kinematically non-condensed (NC) vs condensed (CC) modeling. The main goal is to highlight the effects of kinematic condensation on non-linear modal interactions due to $1: 1 / 2: 1$ internal resonances in shallow horizontal cables as a result of different involvement of symmetric/anti-symmetric modes. Attention is focused on planar dynamics, wherein the effects of the two alternative models already fully display, and the analysis is conducted in such a way to highlight higher-order contributions from both resonant/non-resonant modes to the response of various - crossover/non-crossover - cables. The paper is organized as follows. In Sect. 2, approximate non-linear PDEs of motion of $\mathrm{NC} / \mathrm{CC}$ models are addressed, with a comparison of system quadratic/cubic coefficients obtained through a multi-mode Galerkin expansion. In Sect.3, the asymptotic solution based on second-order multiple scales is summarized for each resonance case. Modal contributions and a comparison of interaction coefficients are discussed in Sect. 4, prior to examining the non-linear response through a continuation technique in Sect. 5. Direct numerical integrations of modulation equations are also performed to verify continuation results. A comparison of space-time varying non-linear dynamic configurations and tensions is carried out in Sect. 6. The paper ends with the conclusions in Sect. 7. 


\section{THEORETICAL BACKGROUND}

A horizontal suspended cable subjected to a uniformly distributed vertical harmonic excitation, $F(x, t)=F \cos \Omega t$, is schematically shown in Fig. 1, with its horizontal span $X_{H}$ and mid-span sag $d$. To attain different sagged configurations, the horizontal component $H$ of cable static tension is varied. The longitudinal (horizontal) and vertical components of planar dynamic displacement, measured from the static equilibrium $y=y(x)$ at an arbitrary cable point, are denoted $u$ and $v$, respectively. The dimensionless variables, $\tilde{x}=\frac{x}{X_{H}}, \tilde{y}=\frac{y}{X_{H}}, \tilde{d}=\frac{d}{X_{H}}, \tilde{u}=\frac{u}{X_{H}}$ $\tilde{v}=\frac{v}{X_{H}}, \tilde{t}=\frac{t}{X_{H}} \sqrt{\frac{g H}{w_{C}}}, \alpha=\frac{E A}{H}, \tilde{c}=c \frac{X_{H}}{H} \sqrt{\frac{g H}{w_{C}}}, \tilde{F}=F \frac{X_{H}}{H}, \tilde{\Omega}=\Omega X_{H} \sqrt{\frac{w_{C}}{g H}}$, and cable parameter $\lambda / \pi=(1 / \pi) \sqrt{\left(w_{C} S_{C}\right)^{2} E A / H^{3}}$ [2] are introduced, with $g$ being the gravity, $E$ the Young's Modulus, $A$ the cross-sectional area, $w_{C}$ the self-weight per unit unstretched length, $S_{C}$ the equilibrium length, $c$ the viscous damping coefficient, $F$ and $\Omega$ the amplitude and frequency of harmonic excitation, respectively. Here, $x$ is the spatial variable, and $t$ denotes time, the prime and dot being the relevant derivatives. The flexible cable is assumed to be homogenous and linear elastic with negligible torsional, bending and shear rigidities. Consider also that the cable has a small initial sag-to-span ratio (less than 1:8) and low static strain, the associated static configuration being described through the parabola $\tilde{y}=4 d \tilde{x}(1-\tilde{x})[2]$. In the following, the $(\sim)$ notation is dropped and zero boundary conditions, i.e., $u(0, t)=u(1, t)=v(0, t)=v(1, t)=0$ are imposed. Symmetric (anti-symmetric) modes correspond to symmetric (anti-symmetric) $v$ and anti-symmetric (symmetric) $u$ components.

\subsection{Kinematically Non-condensed Modeling}

With the assumption of moderately large vibration amplitudes leading to a small dynamic strain, approximate element kinematics of total axial strain $\left(e_{f}\right)$ of cables is given by [5]

$$
e_{f}=e+e_{d}=e+\frac{1}{1+y^{\prime 2}}\left(u^{\prime}+y^{\prime} v^{\prime}+\frac{1}{2}\left(u^{\prime 2}+v^{\prime 2}\right)\right)
$$


where $e(x)$ is the static strain, and $e_{d}(x, t)$ is the dynamic strain expressed through its Lagrangian measure. Due to geometrically non-linear stretching, planar forced damped vibrations of horizontal cables about static equilibrium are governed, in non-dimensional form, by [5]:

$$
\begin{aligned}
& \rho \ddot{u}+c \rho \dot{u}=\left\{u^{\prime}+\frac{\alpha}{\rho^{3}}\left(u^{\prime}+y^{\prime} v^{\prime}\right)+\frac{\alpha}{\rho^{3}}\left(u^{\prime 2}+y^{\prime} u^{\prime} v^{\prime}+\frac{1}{2}\left(u^{\prime 2}+v^{\prime 2}\right)\right)+\frac{\alpha}{2 \rho^{3}}\left(u^{\prime 3}+u^{\prime} v^{\prime 2}\right)\right\}^{\prime}, \\
& \rho \ddot{v}+c \rho \dot{v}=\left\{v^{\prime}+\frac{\alpha}{\rho^{3}}\left(y^{\prime} u^{\prime}+y^{\prime 2} v^{\prime}\right)+\frac{\alpha}{\rho^{3}}\left(u^{\prime} v^{\prime}+y^{\prime} v^{\prime 2}+\frac{y^{\prime}}{2}\left(u^{\prime 2}+v^{\prime 2}\right)\right)+\frac{\alpha}{2 \rho^{3}}\left(u^{\prime 2} v^{\prime}+v^{\prime 3}\right)\right\}^{\prime}+F \rho \cos \Omega t,
\end{aligned}
$$

where $\rho=\sqrt{1+y^{\prime 2}}$. This mechanical model, which holds also for inclined cables $[5,6]$, nonlinearly couples the $u$ and $v$ dynamics, and contains both quadratic/cubic non-linear terms even in the absence of initial sag (i.e., the taut string case). By omitting nonlinearities, damping and forcing terms, natural frequencies and $(u / v)$ modal shapes are determined based on an $N$-terms assumed sine-based series [5]. It is worth noting that, consistent with the assumed parabolic profile, here we put $\rho \approx 1$ in both the linear dynamics analysis and the linear terms of Eqs. (2) and (3), so that the expected differences between $\mathrm{NC} / \mathrm{CC}$ models mainly result from non-linear terms. Yet, higher-order effects of $\rho^{3}$ will be accounted for in non-linear terms since numerical results in [5] have highlighted their significant contribution to non-linear response.

\subsection{Kinematically Condensed Modeling}

For CC model, it is further assumed, with respect to previous assumptions, that (i) the secondorder term $u^{\prime 2}$ of the longitudinal gradient is negligible with respect to unity, and that, besides being $\rho \approx 1$ in statics and linear dynamics, (ii) the higher-order $\rho^{3}$ effects in the non-linear dynamics are also negligible. Furthermore, by assuming that (iii) the cable non-linearly stretches in a quasi-static manner [2] in the absence of longitudinal external loading, the corresponding inertia and viscous damping effects are eliminated. Accounting for Eq. (2) and relevant boundary conditions, the dynamic strain in Eq. (1) becomes

$$
e_{d}(t)=\int_{0}^{1}\left(y^{\prime} v^{\prime}+\frac{1}{2} v^{\prime 2}\right) d x
$$


Thus, the CC model exhibits a space-independent dynamic tension $\left(E A e_{d}\right)$ averaged over the spatial integral, in contrast with Eq. (1) that allows for both spatial and temporal strain variation. Similar to [14-15], planar forced damped dynamics of condensed horizontal cables are governed by a single integro-differential equation associated with $v$ only,

$$
\ddot{v}+c \dot{v}=v^{\prime \prime}+\alpha y^{\prime \prime} \int_{0}^{1} y^{\prime} v^{\prime} d x+\alpha v^{\prime \prime} \int_{0}^{1} y^{\prime} v^{\prime} d x+\frac{\alpha}{2} y^{\prime \prime} \int_{0}^{1} v^{\prime 2} d x+\frac{\alpha}{2} v^{\prime \prime} \int_{0}^{1} v^{\prime 2} d x+F \cos \Omega t,
$$

whereas the $u$ displacement turns out to be $v$-dependent. Closed-form expressions of natural frequencies $(\omega)$ and modes of this CC system can be found in [2, 5].

\subsection{Multi Modal Discretization and Interaction Coefficients}

Eqs. (2) and (3) or Eq. (5) are cast in state-space (first-order) form [7]. Accounting for the orthonormality properties of linear eigenfunctions, the derived equations are projected onto the system full eigenbasis by letting $U^{J}=\sum_{m=1}^{\infty} f_{m} \zeta_{m}^{J}, V^{J}=\sum_{m=1}^{\infty} p_{m} \zeta_{m}^{J}$, in which $J=1,2(J=2)$ for NC (CC) model, $U^{1}=u, U^{2}=v, V^{1}=\dot{u}, V^{2}=\dot{v}, \zeta_{m}^{1}=\phi_{m}, \zeta_{m}^{2}=\varphi_{m}, f_{m}$ and $p_{m}$ being the unknown displacement and velocity coordinates associated with both the longitudinal $\left(\phi_{m}\right)$ and vertical $\left(\varphi_{m}\right)$ shape functions of the $m$ mode [5]. The Galerkin approach is then applied, thereby providing the infinite set of non-linearly coupled ODEs:

$$
\dot{f}_{m}-p_{m}=0, \quad \dot{p}_{m}+2 \mu_{m} p_{m}+\omega_{m}^{2} f_{m}=\sum_{i=1}^{\infty} \sum_{j=1}^{\infty} \Lambda_{m i j} f_{i} f_{j}+\sum_{i=1}^{\infty} \sum_{j=1}^{\infty} \sum_{k=1}^{\infty} \Gamma_{m i j k} f_{i} f_{j} f_{k}+Z_{m} \cos \Omega t,
$$

for $m=1,2, \ldots, \infty$, wherein $2 \mu_{m}=\int_{0}^{1} c\left(\phi_{m}^{2}+\varphi_{m}^{2}\right) d x$ and $Z_{m}=\int_{0}^{1} F \varphi_{m} d x$ are modal damping and forcing terms. With $F$ being a constant, $Z_{m}$ is never (always) zero when $\varphi_{m}$ is a symmetric (antisymmetric) mode. The quadratic and cubic interaction coefficients of NC model are given, respectively, by

$$
\begin{aligned}
& \Lambda_{m i j}=-\alpha \int_{0}^{1} \frac{1}{\rho^{3}}\left\{\phi_{m}^{\prime}\left(\frac{3}{2} \phi_{i}^{\prime} \phi_{j}^{\prime}+y^{\prime} \phi_{i}^{\prime} \varphi_{j}^{\prime}+\frac{1}{2} \varphi_{i}^{\prime} \varphi_{j}^{\prime}\right)+\varphi_{m}^{\prime}\left(\frac{y^{\prime}}{2} \phi_{i}^{\prime} \phi_{j}^{\prime}+\phi_{i}^{\prime} \varphi_{j}^{\prime}+\frac{3}{2} y^{\prime} \varphi_{i}^{\prime} \varphi_{j}^{\prime}\right)\right\} d x, \\
& \Gamma_{m i j k}=-\frac{\alpha}{2} \int_{0}^{1} \frac{1}{\rho^{3}}\left\{\phi_{m}^{\prime}\left(\phi_{i}^{\prime} \phi_{j}^{\prime} \phi_{k}^{\prime}+\phi_{i}^{\prime} \varphi_{j}^{\prime} \varphi_{k}^{\prime}\right)+\varphi_{m}^{\prime}\left(\phi_{i}^{\prime} \phi_{j}^{\prime} \varphi_{k}^{\prime}+\varphi_{i}^{\prime} \varphi_{j}^{\prime} \varphi_{k}^{\prime}\right)\right\} d x,
\end{aligned}
$$

whereas those of CC model read

$$
\Lambda_{m i j}=\alpha\left\{\int_{0}^{1} \varphi_{m} \varphi_{i}^{\prime \prime} d x \int_{0}^{1} y^{\prime} \varphi_{j}^{\prime} d x+\frac{1}{2} \int_{0}^{1} \varphi_{m} y^{\prime \prime} d x \int_{0}^{1} \varphi_{i}^{\prime} \varphi_{j}^{\prime} d x\right\}
$$




$$
\Gamma_{m i j k}=\frac{\alpha}{2}\left\{\int_{0}^{1} \varphi_{m} \varphi_{i}^{\prime \prime} d x \int_{0}^{1} \varphi_{j}^{\prime} \varphi_{k}^{\prime} d x\right\}
$$

Preliminary observation of these physical coefficients provides some hints about possible differences in the associated numerical outcomes [5]:

- Both quadratic/cubic coefficients depend on the mechanical parameter $\alpha$ and the static solution $\left(y^{\prime}, y^{\prime \prime}\right)$, but only the NC model accounts for $\rho^{3}$-term effects.

- The NC (CC) model explicitly accounts for (ignores) non-linear coupling of longitudinal $\phi$ and vertical $\varphi$ displacements, which possibly plays a meaningful role in system response depending on also cable sag and extensibility [18].

- Looking at the terms involving the solely $\varphi$ functions in Eq. (9) or (10) against (7) or (8), it can be seen how the kinematic condensation entails approximating the exact integrals of products of the shape functions of NC model through products of their integrals.

\section{SECOND-ORDER MULTIPLE SCALES SOLUTION}

For a weakly non-linear response, periodic solutions to Eq. (6) are determined using the method of multiple scales (MMS) in condition of primary external and 1:1 or 2:1 internal resonance. The second-order asymptotic analysis is considered, capturing combined effects due to higher-order quadratic and cubic nonlinearities [7]. With $\varepsilon$ denoting a small bookkeeping parameter, we order the damping $\mu_{m}$ and excitation $Z_{m}$ amplitude so that they appear in the same $\varepsilon^{3}$-order problem, in both resonance cases, namely $\mu_{m} \rightarrow \varepsilon^{2} \mu_{m}$ and $Z_{m} \rightarrow \varepsilon^{3} Z_{m}$. The relationships of external and 1:1 internal resonances are quantified through $\Omega=\omega_{s}+\varepsilon^{2} \sigma_{f}, \omega_{s}=\omega_{r}+\varepsilon^{2} \sigma$, whereas those of external and 2:1 internal resonances are expressed as $\Omega=\omega_{s}+\varepsilon \sigma_{f}, \omega_{s}=2 \omega_{r}+\varepsilon \sigma$, with $\sigma_{f}$ and $\sigma$ being external and internal detuning parameters, respectively. In both cases, the external excitation is assumed to put energy into the system via a symmetric $(s)$ mode because $Z_{s} \neq 0$. A companion $r$ mode - which may be either symmetric or anti-symmetric - is driven in the response via relevant internal resonance. Following the MMS analysis of free-undamped 
dynamics in [7] and accounting for modal interaction of the two coupled $(r, s)$ modes, approximate solutions of the forced-damped dynamics are obtained and herein summarized.

\subsection{One-to-One Internal Resonance}

Because 1:1 internal resonance is associated with cubic nonlinearities, the ensuing secular effects appear at $\varepsilon^{3}$ order. The real-valued modulation equations describing the slowly-varying evolution of amplitudes $\left(a_{r}, a_{s}\right)$ and relevant phases $\left(\beta_{r}, \beta_{s}\right)$ are obtained as [18]

$$
\begin{aligned}
& \dot{a}_{r}=-\mu_{r} a_{r}+\frac{K_{1} a_{s}^{3} \sin \Delta}{8 \omega_{r}}+\frac{K_{2} a_{s} a_{r}^{2} \sin \Delta}{8 \omega_{r}}+\frac{K_{3} a_{s}^{2} a_{r} \sin 2 \Delta}{8 \omega_{r}}, \\
& a_{r} \dot{\gamma}_{r}=\left(\sigma_{f}+\sigma\right) a_{r}+\frac{K_{r r} a_{r}^{3}}{8 \omega_{r}}+\frac{K_{r s} a_{r} a_{s}^{2}}{8 \omega_{r}}+\frac{K_{1} a_{s}^{3} \cos \Delta}{8 \omega_{r}}+\frac{3 K_{2} a_{s} a_{r}^{2} \cos \Delta}{8 \omega_{r}}+\frac{K_{3} a_{s}^{2} a_{r} \cos 2 \Delta}{8 \omega_{r}}, \\
& \dot{a}_{s}=-\mu_{s} a_{s}-\frac{K_{2} a_{r}^{3} \sin \Delta}{8 \omega_{s}}-\frac{K_{1} a_{r} a_{s}^{2} \sin \Delta}{8 \omega_{s}}-\frac{K_{3} a_{r}^{2} a_{s} \sin 2 \Delta}{8 \omega_{s}}+\frac{Z_{s} \sin \gamma_{s}}{2 \omega_{s}}, \\
& a_{s} \dot{\gamma}_{s}=\sigma_{f} a_{s}+\frac{K_{s s} a_{s}^{3}}{8 \omega_{s}}+\frac{K_{r s} a_{s} a_{r}^{2}}{8 \omega_{s}}+\frac{K_{2} a_{r}^{3} \cos \Delta}{8 \omega_{s}}+\frac{3 K_{1} a_{r} a_{s}^{2} \cos \Delta}{8 \omega_{s}}+\frac{K_{3} a_{r}^{2} a_{s} \cos 2 \Delta}{8 \omega_{s}}+\frac{Z_{s} \cos \gamma_{s}}{2 \omega_{s}},
\end{aligned}
$$

in which $\gamma_{r}=\left(\sigma_{f}+\sigma\right) t-\beta_{r}, \gamma_{s}=\sigma_{f} t-\beta_{s}, \Delta=\gamma_{r}-\gamma_{s}$, and $K$ are second-order coefficients depending on the infinite-dimensional modal series and governing quadratic and cubic contributions. They read:

$$
\begin{aligned}
K_{h h}= & \sum_{m=1}^{\infty}\left[\left(\Lambda_{h h m}+\Lambda_{h m h}\right) \Lambda_{m h h}\left(\frac{2}{\omega_{m}^{2}}+\frac{1}{\omega_{m}^{2}-4 \omega_{h}^{2}}\right)\right]+3 \Gamma_{h h h h}, h=r, s \\
K_{r s}= & \sum_{m=1}^{\infty}\left[\left(\Lambda_{r r m}+\Lambda_{r m r}\right) \frac{2 \Lambda_{m s s}}{\omega_{m}^{2}}+\left(\Lambda_{r s m}+\Lambda_{r m s}\right)\left(\Lambda_{m r s}+\Lambda_{m s r}\right)\left(\frac{1}{\omega_{m}^{2}-\left(\omega_{s}+\omega_{r}\right)^{2}}+\frac{1}{\omega_{m}^{2}-\left(\omega_{s}-\omega_{r}\right)^{2}}\right)\right] \\
& +2\left(\Gamma_{r s s r}+\Gamma_{r s r s}+\Gamma_{r r s s}\right), \\
K_{1}= & \sum_{m=1}^{\infty}\left[\left(\Lambda_{s r m}+\Lambda_{s m r}\right) \frac{\Lambda_{m s s}}{\omega_{m}^{2}-4 \omega_{s}^{2}}+\frac{\left(\Lambda_{s s m}+\Lambda_{s m s}\right)\left(\Lambda_{m r s}+\Lambda_{m s r}\right)}{\omega_{m}^{2}-\left(\omega_{s}-\omega_{r}\right)^{2}}\right]+\Gamma_{s s s r}+\Gamma_{s s r s}+\Gamma_{s r s s}, \\
K_{2}= & \sum_{m=1}^{\infty}\left[\left(\Lambda_{s r m}+\Lambda_{s m r}\right) \Lambda_{m r r}\left(\frac{2}{\omega_{m}^{2}}+\frac{1}{\omega_{m}^{2}-4 \omega_{r}^{2}}\right)\right]+3 \Gamma_{s r r r}, \\
K_{3}= & \sum_{m=1}^{\infty}\left[\left(\Lambda_{s s m}+\Lambda_{s m s}\right) \frac{\Lambda_{m r r}}{\omega_{m}^{2}-4 \omega_{r}^{2}}+\frac{\left(\Lambda_{s r m}+\Lambda_{s m r}\right)\left(\Lambda_{m r s}+\Lambda_{m s r}\right)}{\omega_{m}^{2}-\left(\omega_{s}-\omega_{r}\right)^{2}}\right]+\Gamma_{s r r s}+\Gamma_{s r s r}+\Gamma_{s s r r} .
\end{aligned}
$$

Note that the 1:1 resonant interaction is activated only if at least one of $K_{1}, K_{2}$ and $K_{3}$ is nontrivial [8]. Based on the assumed full-basis Galerkin expansion, the second-order coupled forced 
dynamic configurations associated with the $u(J=1)$ or $v(J=2)$ displacement component of a 1:1 resonant suspended cable are expressed as

$$
\begin{aligned}
U^{J}(x, t) & \approx a_{r} \cos \left(\Omega t-\gamma_{r}\right) \zeta_{r}^{J}(x)+a_{s} \cos \left(\Omega t-\gamma_{s}\right) \zeta_{s}^{J}(x)+ \\
& \frac{1}{2}\left\{\begin{array}{l}
a_{s}^{2}\left[\cos \left(2 \Omega t-2 \gamma_{s}\right) \psi_{s s}^{J}(x)+\kappa_{s s}^{J}(x)\right]+a_{r}^{2}\left[\cos \left(2 \Omega t-2 \gamma_{r}\right) \psi_{r r}^{J}(x)+\kappa_{r r}^{J}(x)\right]+ \\
a_{s} a_{r}\left[\cos \left(2 \Omega t-\gamma_{r}-\gamma_{s}\right) \psi_{r s}^{J}(x)+\cos \left(\gamma_{r}-\gamma_{s}\right) \kappa_{r s}^{J}(x)\right]
\end{array}\right\},
\end{aligned}
$$

with $J=1,2(J=2)$ for NC (CC) model. The pertinent spatial corrections $\psi_{i j}^{J}, \kappa_{i j}^{J}$ accounting for quadratic effects of all infinite modes can be found in [7].

\subsection{Two-to-One Internal Resonance}

The secular effects due to a 2:1 internal resonance appear at $\varepsilon^{2}$ order which is associated with quadratic nonlinearities only. However, one has to account for also the higher $\varepsilon^{3}$-order effects associated with both quadratic and cubic nonlinearities so as to refine the asymptotic-based solution [6]. We consider primary resonance of a high-frequency symmetric s mode, instead of a low-frequency $r$ one which may be either symmetric or anti-symmetric. The relevant real-valued modulation equations read [18]

$$
\begin{aligned}
& \dot{a}_{r}=-\mu_{r} a_{r}+\frac{\mathfrak{R} a_{r} a_{s} \sin \gamma_{r}}{4 \omega_{r}}, \\
& a_{r}\left(\dot{\gamma}_{r}+\dot{\gamma}_{s}\right)=\left(\sigma_{f}+\sigma\right) a_{r}+\frac{\mathfrak{R} a_{r} a_{s} \cos \gamma_{r}}{2 \omega_{r}}+\frac{K_{r r} a_{r}^{3}}{4 \omega_{r}}+\frac{K_{r s} a_{r} a_{s}^{2}}{4 \omega_{r}}, \\
& \dot{a}_{s}=-\mu_{s} a_{s}-\frac{\mathfrak{R} a_{r}^{2} \sin \gamma_{r}}{8 \omega_{s}}+\frac{Z_{s} \sin \gamma_{s}}{2 \omega_{s}}, \\
& a_{s} \dot{\gamma}_{s}=\sigma_{f} a_{s}+\frac{\Re a_{r}^{2} \cos \gamma_{r}}{8 \omega_{s}}+\frac{K_{s s} a_{s}^{3}}{8 \omega_{s}}+\frac{K_{r s} a_{s} a_{r}^{2}}{8 \omega_{s}}+\frac{Z_{s} \cos \gamma_{s}}{2 \omega_{s}}
\end{aligned}
$$

where $\gamma_{r}=\sigma t-2 \beta_{r}+\beta_{s}, \gamma_{s}=\sigma_{f} t-\beta_{s}$, and the first-order interaction coefficient $\mathfrak{R}=2 \Lambda_{s r r}[5,7]$.

The second-order coefficient $K_{s s}$ is identical to Eq. (15) of 1:1 resonance case, whereas

$$
K_{r r}=\frac{9}{4 \omega_{s}^{2}} \Lambda_{s r r}\left(\Lambda_{r r s}+\Lambda_{r s r}\right)+\sum_{\substack{m=1, m \neq s}}^{\infty}\left[\left(\Lambda_{r r m}+\Lambda_{r m r}\right) \Lambda_{m r r}\left(\frac{2}{\omega_{m}^{2}}+\frac{1}{\omega_{m}^{2}-4 \omega_{r}^{2}}\right)\right]+3 \Gamma_{r r r r},
$$




$$
\begin{aligned}
K_{r s}= & \sum_{\substack{m=1, m \neq r}}^{\infty}\left[\left(\Lambda_{r r m}+\Lambda_{r m r}\right) \frac{2 \Lambda_{m s s}}{\omega_{m}^{2}}+\left(\Lambda_{r s m}+\Lambda_{r m s}\right)\left(\Lambda_{m r s}+\Lambda_{m s r}\right)\left(\frac{1}{\omega_{m}^{2}-\left(\omega_{s}+\omega_{r}\right)^{2}}+\frac{1}{\omega_{m}^{2}-\left(\omega_{s}-\omega_{r}\right)^{2}}\right)\right] \\
& +\frac{\left(\Lambda_{r r s}+\Lambda_{r s r}\right)^{2}}{8 \omega_{r}^{2}}+\frac{4}{\omega_{r}^{2}} \Lambda_{r s s} \Lambda_{r r r}+2\left(\Gamma_{r s s r}+\Gamma_{r s r s}+\Gamma_{r r s s}\right) .
\end{aligned}
$$

The 2:1 resonant interaction is activated only if $\mathfrak{R}$ is non-trivial $[6,8]$, a circumstance which herein certainly occurs because the high-frequency mode is symmetric. In turn, the second-order coupled forced dynamic configurations of a 2:1 resonant suspended cable read

$$
\begin{aligned}
& U^{J}(x, t) \approx a_{r} \cos \left(\frac{\Omega t}{2}-\frac{\gamma_{s}}{2}-\frac{\gamma_{r}}{2}\right) \zeta_{r}^{J}(x)+a_{s} \cos \left(\Omega t-\gamma_{s}\right) \zeta_{s}^{J}(x)+ \\
& \frac{1}{2}\left\{\begin{array}{l}
\left.a_{s}^{2}\left[\cos \left(2 \Omega t-2 \gamma_{s}\right) \psi_{s s}^{J}(x)+\kappa_{s s}^{J}(x)\right]+a_{r}^{2}\left[\cos \left(\Omega t-\gamma_{s}-\gamma_{r}\right) \psi_{r r}^{J}(x)+\kappa_{r r}^{J}(x)\right]+\right] \\
a_{s} a_{r}\left[\cos \left(\frac{3}{2} \Omega t-\frac{3}{2} \gamma_{s}-\frac{1}{2} \gamma_{r}\right) \psi_{r s}^{J}(x)+\cos \left(\frac{1}{2} \Omega t-\frac{1}{2} \gamma_{s}+\frac{1}{2} \gamma_{r}\right) \kappa_{r s}^{J}(x)\right]
\end{array}\right\},
\end{aligned}
$$

where the spatial shape functions $\left(\psi_{i j}^{J}, \kappa_{i j}^{J}\right)$ can be found in $[5,7]$.

Mention must be made that, for the sake of generality, we account for also higher-order effects of $\sigma$ both in second-order coefficients, Eqs. (16), (17), (19) and (26), of modulation equations, and in spatial displacement corrections of Eqs. (20) and (27), whereas these effects were disregarded in $[5,6]$. On accounting for higher-order quadratic effects from every mode in the coupled amplitudes and displacements, the major drawback of discretization solution - in which the spatial variation in non-linear problem is assumed to be the same as that in linear problem - has been overcome. Indeed, it is seen from Eqs. (20) and (27) how, in NC (CC) model, both (only) $u$ and $v(v)$ are (is) spatially influenced by contributions from all of the modal eigenfunctions. This occurs irrespective of the fact that the amplitudes obtained with the two models might be already different due to their different coefficients (see Sects. 2.3, 4 and 5). Thus, it is conjectured that overall errors in the associated non-linear dynamics may be meaningful. In the following, a prerequisite analysis of interaction coefficients is presented. 


\section{ANALYSIS OF 1:1/2:1 RESONANT INTERACTION COEFFICIENTS}

Planar 1:1 resonances involving mixed (symmetric/anti-symmetric) modes may occur near crossovers $(\lambda / \pi \approx 2 n, n=1,2, \ldots)$ in the frequency spectrum of Fig. 2, which displays, based on $\mathrm{NC}$ model, the first six planar frequencies $\omega / \pi$ (dimensionalized with respect to the fundamental frequency of the corresponding taut string) versus $\lambda / \pi$. In turn, planar 2:1 resonances may occur either near (e.g., $\lambda / \pi \approx 4$ ) or away from (e.g., $\lambda \pi \approx 1.28,2.95,5.48$ ) crossovers, involving either mixed or purely symmetric modes [6, 8], respectively. Here, out-of-plane displacement contributions entering a multiple internal resonance are not taken into account. The aim of the analysis is twofold: (i) to distinguish contributions from different resonant/non-resonant modes to second-order quadratic coefficients of 1:1/2:1 or crossover/non-crossover resonant cables; (ii) to make a comparison of effective coefficients for some chosen $\mathrm{NC} / \mathrm{CC}$ cables having the same elasto-geometric properties $\left(\alpha, d, X_{H}, w_{C}\right)$.

Three resonant cables are considered, whose properties based on NC model are summarized in Table 1, with the fixed values $X_{H}=850 \mathrm{~m}, A \approx 0.1159 \mathrm{~m}^{2}, w_{C} \approx 9.48 \mathrm{kN} / \mathrm{m}$ and $E=1.794 \times 10^{8}$ $\mathrm{kN} / \mathrm{m}^{2}[3,5-6]$. Their high-frequency $s$ modes are symmetric, and $d$ values are less than 1:8 [2]. The non-crossover $(\lambda / \pi \approx 2.95)$ cable involves symmetric $(s, r)$ modes [6], whereas the crossover $(\lambda / \pi \approx 2.02,4.03)$ cables involve, though being different depending on the activated internal resonance, anti-symmetric low-frequency $r$ modes, see also $v$ shapes in Fig. 2. The convergent number $N$ of the assumed sine series in linear dynamics increases with $d$ and $\alpha$, and the modal integral $\Upsilon_{s}$ affecting the forcing magnitude $\left(Z_{s}=F \Upsilon_{s}\right)$ changes with the solely $v$ shape function.

\subsection{Modal Interaction Perspectives}

Actual features of internal resonance activation and possible solutions are drawn as follows.

I. Nearly $\left(\omega_{m} \approx 2 \omega_{r} \approx 2 \omega_{s}\right)$, instead of perfectly, tuned cables are considered in Table 1, to avoid singularities due to small divisor terms in Eqs. (15)-(19) or (25)-(26), i.e., $\omega_{m}^{2}-\left(\omega_{s}+\omega_{r}\right)^{2}$ or $\omega_{m}^{2}-4 \omega_{h}^{2}, h=r$ or $s$. 
II. The NC model involving a 1:1 resonance exhibits - though being not explicit - vanishing $K_{1}$ and $K_{2}$ through the embedded quadratic (Eq.7) and cubic (Eq.8) coefficients, in agreement with CC model through Eqs. (9) and (10) [8, 18]. This is because the non-linear orthogonality properties hold between the eigenfunctions of mixed modal types at crossovers. With $m$ being the anti-symmetric (symmetric) resonant or non-resonant mode, the ensuing quadratic coefficients $\Lambda_{m s s}, \Lambda_{s s m}, \Lambda_{s m s}\left(\Lambda_{s r m}, \Lambda_{s m r}, \Lambda_{m r s}, \Lambda_{m s r}\right)$ and $\Lambda_{m r r}\left(\Lambda_{s r m}, \Lambda_{s m r}\right)$ of both models are identically zero in $K_{1}$ and $K_{2}$, respectively, and all of the associated cubic coefficients $\Gamma_{s s s r}, \Gamma_{s s r s}, \Gamma_{s r s s}$ and $\Gamma_{s r r r}$ are zero in both $K_{1}$ and $K_{2}$, too. In contrast, $K_{3}$ is always non-trivial, and the $1: 1$ resonance seems to be always activated at crossovers [8].

III. As regards the 2:1 resonance, both $\mathrm{NC}$ and $\mathrm{CC}$ models never reveal zero values of $\mathfrak{R}$ when the symmetric high-frequency mode is involved in the internal resonance. Thus, such 2:1 resonance is always activated in horizontal cables $[6,8]$.

IV. Analysis of Eqs. (11)-(14) or (21)-(24) reveals two kinds of possible steady-state response $(\dot{a}=\dot{\gamma}=0)$, namely the uncoupled-mode $\left(a_{r}=0, a_{s} \neq 0\right)$ solution of the directly excited symmetric mode, and the coupled-mode $\left(a_{r} \neq 0, a_{s} \neq 0\right)$ solution. However, while in the $1: 1$ resonance the uncoupled solution occurs regardless of the low- or high-frequency mode being excited (i.e., $\sigma$ may be positive or negative) only due to the vanishing $K_{1}$ and $K_{2}$, it always (never) exists in the 2:1 resonance involving the high-frequency (low-frequency) excited mode.

\subsection{Second-Order Quadratic Modal Contributions}

Because both NC/CC models exhibit similar modal participation with the same number of retained modes [18], we use the more general $\mathrm{NC}$ model to discuss the modal contributions to second-order quadratic (a superscript $q$ ) coefficients due to a $1: 1\left(K_{i i}^{q}, K_{3}^{q}\right)$, in comparison with a 2:1 $\left(K_{i i}^{q}\right)$, resonance. Accounting for the first 10 modes of $1: 1$ resonant first-crossover $(\lambda / \pi \approx$ 2.02 ) and $2: 1$ resonant non-crossover cables $(\lambda / \pi \approx 2.95)$, the pertinent percent contributions to 
each of the coefficients are reported in Table 2, which distinguishes between resonant (underlined) and non-resonant, as well as symmetric (S) and anti-symmetric (A), modes. For the 1:1 resonant cable, both symmetric and anti-symmetric (low-order) modes contribute to $K_{r s}^{q}, K_{3}^{q}$, whereas only symmetric modes contribute to $K_{r r}^{q}, K_{s s}^{q}$. In accordance with Sect.4.1 (II), these differences occur because of the non-linear orthogonality: the two resonant $(s, r)$ mixed $(\mathrm{S}, \mathrm{A})$ modal-based functions (e.g., $\Lambda_{m r s}$ ) substantially affect $K_{r s}^{q}$ and $K_{3}^{q}$ in Eqs.(16) and (19), respectively, whereas only purely based - either $s$ or $r\left(\mathrm{~S}\right.$ or A) - modal functions (e.g., $\Lambda_{h h m}, h$ $=r$ or $s$ ) appear in Eq. (15) of $K_{r r}^{q}$ and $K_{s s}^{q}$. In turn, such orthogonality allows for solely nonvanishing symmetric modal contributions in the $2: 1$ resonance case $(\lambda / \pi \approx 2.95)$, see also [6] for $\lambda / \pi \approx 1.28$ and 5.48 . To sustain these aspects, the larger-sagged cable at second $\operatorname{crossover}(\lambda / \pi \approx$ 4.03) - which may exhibit either $1: 1$ or $2: 1$ resonance - is considered in Table 3 , by accounting for the first 15 modes. Because of involving the same high-frequency mode $(s=4)$ and no antisymmetric contribution, the symmetric modal contributions to $K_{s s}^{q}$ are practically identical in both resonance cases. Besides the role played by a higher (order) number of symmetric nonresonant modes due to the increased sag effect, it can now be seen that anti-symmetric modes come into play when the cable exhibits not only $1: 1\left(K_{r s}^{q}, K_{3}^{q}\right)$ but also 2:1 (i.e., $\left.K_{r s}^{q}\right)$ resonance, because of involving mixed resonant S/A modes.

In view of Table 2 or 3 , anti-symmetric modal contributions of resonant $\left(1^{\text {st }}\right.$ or $\left.3^{\text {rd }}\right)$ mode are much higher than the negligible ones of non-resonant modes. It is also noticed (Table 3) that, when the $1: 1(2: 1)$ resonance involving the anti-symmetric $3^{\text {rd }}\left(1^{\text {st }}\right)$ mode is activated, even the contributions from the anti-symmetric low-frequency (high-frequency) $1^{\text {st }}\left(3^{\text {rd }}\right)$ mode - which might be involved in the coexisting 2:1 (1:1) resonance - are negligible. Thus, one may discard $a$ priori anti-symmetric non-resonant modes from the relevant discretization. This makes possible to avoid the expected singularity of the MMS solution for a perfectly tuned cable, see Sect. 4.1 
(I). For a proper reduced-order model selection, it is suggested accounting for up to the $5^{\text {th }}$ mode for smaller-sagged cables (Table 2), but up to the $9^{\text {th }}$ mode for larger-sagged cables (Table 3 ).

Overall, the second-order quadratic modal contributions due to $1: 1$ or $2: 1$ resonances at crossovers are in agreement with those due to 2:1 resonances away from crossovers [6], thus confirming the importance of accounting for both resonant and non-resonant (higher-order, mostly symmetric) modes in the asymptotic solution of even shallow cables. This is always valid unless a very shallow cable (e.g., $\lambda / \pi \approx 1.28)$ is considered, wherein non-resonant modes are negligible [6]. To get solution convergence, the non-resonant modes become as more important as the cable sag is increased towards higher $\lambda / \pi$ values (e.g., $\lambda / \pi \approx 5.48$ ), which require more retained modes. By way of example, non-resonant modal contributions to $K_{r r}^{q}, K_{s s}^{q}, K_{r s}^{q}$ are about 7.03, 7.55 and $-10.76 \%$ for the first-crossover cable (Table 2), whereas they become 26.39, 28.86 and $-30.98 \%$ for the second-crossover cable (Table 3), respectively. Such meaningful effects have been overlooked in several papers that investigated modal interactions near crossovers by accounting for only resonant modes in the second-order analysis or by constraining the analysis to just the first order [1]. Based on the MMS, the lowest-dimensional discretization yields quantitatively-inaccurate and/or qualitatively-crude results with respect to the coinciding infinite-dimensional discretization [6] or direct $[12,14]$ perturbation.

\subsection{A Comparison of Effective Non-linear Coefficients}

The effective first- $(\mathfrak{R})$ and second-order $(K)$ coefficients of $\mathrm{NC} / \mathrm{CC}$ cables in Tables 2 and 3 are now compared in Tables $4 \mathrm{a}$ and $4 \mathrm{~b}$, respectively. Apart from overall significant quantitative discrepancies, it can be seen that, for 1:1 resonant first (Table 4a) and second (Table 4b) crossover cables, there is no sign difference in all of the $K_{r r}, K_{s s}, K_{r s}, K_{3}$ values; in particular, the CC model predicts smaller (larger) absolute values of $K_{r r}\left(K_{s s}, K_{r s}, K_{3}\right)$, thus entailing weaker hardening effects [7]. As regards 2:1 resonant cables, the CC model predicts larger absolute values of $\mathfrak{R}$. Moreover, while only - though substantial - quantitative difference is amenable to 
$K_{r r}$ and $K_{s s}$, a remarkable sign difference occurs in $K_{r s}$ for both non-crossover (Table 4a) and second-crossover (Table 4b) cables. As discussed in [5, 18], such circumstance occurs because in $K_{r s}$ the difference of the embedded cubic coefficients between NC/CC models is substantially larger than that of the embedded quadratic coefficients. The underlying physical meaning is that, based on second-order analysis, the $\mathrm{CC}$ model reduces (strengthens) the degree of hardening (softening) nonlinearities, since meaningful higher-order effects of longitudinal dynamic deformation due to cable stretching are neglected through the kinematic condensation. Such remarkable differences in overall values and/or sign of some coefficients are capable of influencing the ensuing non-linear dynamics and bifurcations of the two cable models.

\section{A COMPARISON OF NONLINEAR DYNAMIC RESPONSES}

A $\mathrm{NC} / \mathrm{CC}$ comparison of non-linear dynamic responses is made by means of frequency- and force-response diagrams. To evaluate the equilibrium (fixed points) and dynamic solutions, the Cartesian form of Eqs. (11)-(14) or (21)-(24) is considered. Following [19], the fixed points representing steady-state motion are evaluated by the Newton-Raphson procedure, whereas the dynamic solutions representing limit cycles are evaluated based on the shooting application, with overall paths being traced out, upon varying a control parameter, via the continuation approach. Stability of fixed points is analyzed based on eigenvalues of the relevant $4 \times 4$ Jacobian matrix, whereas stability of limit cycles is evaluated based on Floquet multipliers. As mentioned in Sect. 4.1 (IV), both coupled $\left(a_{s}-a_{r}\right)$ and uncoupled $\left(a_{s}\right)$ solutions exist in both 1:1/2:1 resonances. For the sake of comparison, we choose small damping $c$ such that $\mu_{r}=.005$ and $\mu_{s}=.006$, and consider perfect tuning $\sigma=0$. These parameters are kept constant in all cases. In the following, solid lines indicate stable fixed points, whereas dashed and dotted lines indicate unstable fixed points whose stability is exchanged through saddle-node (SN) or pitchfork (PF) and Hopf (HF) bifurcations, respectively. Filled (open) circles indicate stable (unstable) limit cycles. 


\subsection{First-Crossover Resonant Cable}

The first-crossover cable, commonly considered in the literature [1], is first analyzed in the 1:1 resonant interaction case. The first comparison is made through the frequency-response curves shown in Fig. 3 with a small forcing amplitude $F=.002\left(Z_{s} \approx .0016\right)$. The qualitative resemblance of overall pictures and bifurcation types with NC (Fig. 3a) and CC (Fig. 3b) models is remarkable: both exhibit the stable/unstable coupled solution $\left(a_{s}-a_{r}\right)$ curves on both sides of the zero- $\sigma_{f}$ axis, which ensue from the uncoupled $a_{s}$ branches via $\mathrm{PF}_{1}$ and $\mathrm{PF}_{2}$ bifurcations. In particular, both diagrams exhibit softening-type nonlinearities, and the stable coupled solutions persist over a wide $\sigma_{f}$ range. The directly (internally-resonant) excited $a_{s}\left(a_{r}\right)$ amplitude prevails left (right) of perfect external tuning $\left(\sigma_{f}=0\right)$. These dynamic characteristics are in good qualitative agreement with the responses in [14], where, however, also multi-modal interactions of $\mathrm{CC}$ cables due to multiple internal resonances are considered, thus highlighting a more complex dynamics and bifurcation scenario in a certain $\sigma_{f}$ range.

Small quantitative differences occur between Figs. 3a and 3b, being appreciable mostly as regards the large-amplitude coupled branches left to the zero- $\sigma_{f}$ axis. The $\mathrm{CC}$ model underestimates $a_{s}$ and slightly overestimates $a_{r}$, by affecting the former greater than the latter. The overall distinctive outcomes are due to the fact that, with the two models, the difference in the uncoupled branch is solely controlled by the different coefficient $K_{s s}$, whereas the difference in the coupled branch is thoroughly affected by all of the different coefficients $K_{r r}, K_{s s}, K_{r s}$ and $K_{3}$ (see Eqs. 11-14). As a result, the CC model exhibits more softening non-linear response than the $\mathrm{NC}$ one, as already discussed in Table 4a. In addition, all of the associated bifurcations $\left(\mathrm{SN}_{1}\right.$, $\mathrm{PF}_{1}, \mathrm{PF}_{2}$ ) slightly shift to the left.

It is of interest to compare and discuss the force-response diagrams, so as to realize how much the discrepancies evolve when varying the forcing amplitude $F$. The ensuing NC (CC) results are depicted in Figs.4a (4b) and 4c (4d) for the given detunings $\sigma_{f}=-.12$ and .12 , respectively. Depending on the initial conditions, multiple steady-state solutions coexist in both $\mathrm{NC} / \mathrm{CC}$ 
diagrams, which exhibit, at most, five (one stable and two unstable uncoupled, one stable and one unstable coupled) or three (one stable uncoupled, one stable and one unstable, or two unstable, coupled) fixed points in Figs. 4a, b or 4c, d respectively. The corresponding uncoupled solutions lose and gain their stability via $\mathrm{PF}_{1}$ and $\mathrm{PF}_{2}$ bifurcations at low and higher $F$ in Figs.4a $(F \approx .00296, .01720)$ and $4 \mathrm{~b}(F \approx .002645, .00825)$, whereas they remain stable and isolated from the coupled solutions over the whole considered $F$ range in Figs. $4 c$ and $4 d$, respectively.

Overall, quantitative discrepancies in force-response curves and bifurcation locations between $\mathrm{NC} / \mathrm{CC}$ models are observable, especially in the predominantly stable coupled solutions, and the differences become more remarkable when increasing $F$. While Figs. $4 \mathrm{a}$ and $4 \mathrm{~b}$ look substantially similar, there are significant qualitative differences between Figs.4c and $4 \mathrm{~d}$ as regards bifurcation features. Indeed, the NC model (Fig. 4c) shows the occurrence of a large region of dynamic solutions in between the two Hopf bifurcations at $F \approx .0161\left(\mathrm{HF}_{1}\right)$ and 0.0482 $\left(\mathrm{HF}_{2}\right)$ in the stable coupled branches, whereas the $\mathrm{CC}$ model (Fig. $4 \mathrm{~d}$ ) reveals none of them. Similar to Fig. 3, the CC model underestimates (overestimates) $a_{s}\left(a_{r}\right)$ values with respect to the NC one. As a result, in the considered $F$ range, the stable coupled $a_{r}$ amplitudes in Fig. $4 \mathrm{~d}$ are seen to be always greater than the corresponding $a_{s}$ amplitudes, in contrast with Fig. 4c, where $a_{s}$ dominates the coupled response in the larger $F$ range.

\subsection{Second-Crossover Resonant Cable}

A comparison of frequency-response diagrams of 1:1 resonant second-crossover cable is presented in Fig. 5. In this region, using the CC model, Pakdemirli et al. [12] also investigated a 1:1 resonance, however involving in-plane/out-of-plane modes, whereas a NC/CC comparison of responses due to planar 2:1 resonance is reported in [18]. With the same assigned parameters and $\sigma_{f}$ range as of the first-crossover cable, the softening dynamic responses of NC (Fig. 5a) and CC

(Fig. 5b) models are still in qualitative agreement, and they are similar to those in Figs. 3a and $3 \mathrm{~b}$ because the sign of $K_{r r}, K_{s s}, K_{r s}$ and $K_{3}$ does not change for the higher crossover cable (Table $4 \mathrm{~b}$ 
vs 4a). However, overall response in Fig. 5 is more softening, as expected due to the larger sag. Again, the $\mathrm{CC}$ model predicts more softening behavior than the $\mathrm{NC}$ one.

As regards stability and bifurcations, some remarkable changes occur in both models with respect to Fig. 3. First, there are two Hopf bifurcations in the coupled branches before they experience the jump via $\mathrm{SN}_{1}$ to the uncoupled branches, when $\sigma_{f}$ is swept up. The $\mathrm{HF}_{1}$ and $\mathrm{HF}_{2}$ points occurring around $\sigma_{f}=0$ are responsible for coupled-mode periodic solution. Second, the $\mathrm{PF}_{2}$ bifurcation evidently shifts to lower $\sigma_{f}$ values. It is also worth remarking that the range of coupled solution (between $\mathrm{SN}_{2}-\mathrm{PF}_{1}$ in Fig.5), where there is no coexisting stable uncoupled solution, is greater than that of the first crossover cable. Moreover, some major extent of energy transfer from the directly excited $a_{s}$ mode to the driven $a_{r}$ mode is seen to occur. A comparison of dynamic solution branches emerged from $\mathrm{HF}_{1}$ and $\mathrm{HF}_{2}$ with $\mathrm{NC} / \mathrm{CC}$ models is depicted in Figs.5c and 5d. Apart from being the $\sigma_{f}$ ranges of occurrence slightly shifted with respect to each other, both models predicts a sub-critical (super-critical) bifurcation $\mathrm{HF}_{1}\left(\mathrm{HF}_{2}\right)$ because the associated born limit cycle is unstable (stable), and a jump phenomenon of the stable branch onto the steady response via cyclic-fold bifurcation when sweeping $\sigma_{f}$ down.

To verify the existence of amplitude-steady or -modulated solutions and to better characterize the modal interactions, as distinguished by the stability, the continuation $\mathrm{NC} / \mathrm{CC}$ results are complemented by numerical integrations of modulation equations. The fourth-order Runge-Kutta scheme is considered, with initial conditions of the NC/CC fixed points. Associated with Figs. 5a and $5 \mathrm{~b}$ in which $F=.002$ and $\sigma=0$, the time histories showing the coupled $a_{r}$ (solid lines) and $a_{s}$ (dashed lines) amplitudes of NC and CC cables are illustrated in Figs. 6a and $6 \mathrm{~b}$ for $\sigma_{f}=-0.1$, and in Figs. 6c and 6d for $\sigma_{f}=0$, respectively. Considering still $\sigma_{f}=0$ and $\sigma=0$, but a larger $F$ (.005), the associated time histories are shown in Figs. 6e (NC) and $6 \mathrm{f}(\mathrm{CC})$. A convergent time step equal to .0001 is considered in all cases. 
It can be seen that, after experiencing initial short-interval transient dynamics, both NC (Fig.6a) and CC (Fig.6b) time responses settle down onto steady-state coupled responses, as predicted by the continuation for $\sigma_{f}=-0.1$. Analogously, for $\sigma_{f}=0$, the predicted coupled-mode dynamic solutions after Hopf bifurcations (Figs.5a and 5b) are confirmed by the periodically amplitude-modulated time histories established in Figs.6c and 6d upon imposing the unstable fixed-point $\mathrm{NC}$ and $\mathrm{CC}$ solutions as initial conditions. Thus, the $\mathrm{NC}$ and $\mathrm{CC}$ temporal laws are in agreement - though showing some quantitative differences - as regards stationary and nonstationary amplitude response features. However, for the same second-crossover cable, when increasing $F$ while keeping other parameters and initial conditions unchanged, more marked amplitude-dependent limit cycles and stronger interaction features are seen in Figs.6e and $6 \mathrm{f}$ with respect to $6 \mathrm{c}$ and $6 \mathrm{~d}$, respectively. Essentially, qualitative differences - in terms of both the extent and duration of interaction - occur between NC (Fig.6e) and CC (Fig.6f) models according to the fact that the associated dynamic amplitudes are substantially different at $\sigma_{f}=0$ (Figs. 5c vs 5d). Overall, the numerical results validate the continuation outcomes, yet providing a further comparison in terms of non-linear temporal behaviors.

\subsection{Non-Crossover Resonant Cable}

Moving to the non-crossover cable $(\lambda / \pi \approx 2.95)$ exhibiting $2: 1$ resonance, major quantitative and qualitative discrepancies are visible between the frequency-response curves of NC (Fig.7a) and CC (Fig.7b) models. The uncoupled $a_{s}$ solution now exhibits a hardening non-linearity and the indirectly-excited $a_{r}$ amplitudes play a dominant - or comparable - role in the coupled responses with respect to the directly-excited $a_{s}$ amplitudes. The differences between the two models are significant mostly as regards the coupled branches emanated from the uncoupled branches via $\mathrm{PF}_{1}$ and $\mathrm{PF}_{2}$ bifurcations. This occurs even though the overall forcing magnitude is reduced, with respect to the crossover cable cases, to $Z_{s} \approx .0005$, while fixing $F=.002$, due to a decreased $\Upsilon_{s}$ (Table 1). Remarkably, the CC model (Fig.7b) predicts overestimated $a_{r}$ values, 
with the associated coupled responses being confined in a marginal $\sigma_{f}$ range with respect to NC model (Fig.7a). Moreover, the former reveals the existence of dynamic solutions with two $\mathrm{HF}_{1} / \mathrm{HF}_{2}$ bifurcations, which do not occur in the latter.

Apart from the very large quantitative errors, the qualitative differences of $\mathrm{NC}$ and $\mathrm{CC}$ models are definitely apparent in the force-response diagrams of Fig. 8 , with $\sigma_{f}=0$ corresponding to Fig.7. The NC coupled and uncoupled branches in Fig.8a are seen to be isolated from each other in the considered $F$ range, with the latter being stable over the entire interval. On the other hand, the $\mathrm{CC}$ model reveals in Fig.8b how the uncoupled branch loses stability via $\mathrm{PF}_{1}$ at a very low $F$ and regains stability at $\mathrm{PF}_{2}$. The stable coupled $a_{s}$ branch is almost coincident with the unstable uncoupled one. This shows that the stable coupled solution takes part in only a certain $F$ range, unlike Fig.8a, where the stable coupled/uncoupled branches nearly always coexist with their amplitudes monotonously increasing when increasing $F$. These outstanding qualitative differences may be attributed - besides to the overall quantitative differences in the first- and second-order coefficients governing the amplitudes at different orders - also to the sign difference in the resulting $K_{r s}$ of the two models discussed in Sect. 4.3.

\section{A COMPARISON OF NONLINEAR DYNAMIC DISPLACEMENTS AND TENSIONS}

A comparison of non-linear dynamic displacements furnished by $\mathrm{NC} / \mathrm{CC}$ models is now presented, in conjunction with the significance of space-time non-linear dynamic tensions [3, 4, 6], which has been overlooked in many studies. Based on the obtained stable amplitudes, the 1:1 or 2:1 resonant coupled dynamic configurations accounting for second-order spatial corrections are constructed through Eq. (20) or (27), respectively. The NC dynamic tensions $\left(T_{d}\right)$ accounting for both $u$ and $v$ displacements are evaluated through the dynamic strain $e_{d}$ in Eq. (1), whereas the approximate - spatially constant - CC dynamic tensions are evaluated based on Eq. (4). In all cases, 51 cable nodes from the left support are considered. 
Coupled $v$ displacements of 1:1 resonant first-crossover $\left(\sigma=\sigma_{f}=0, F=.002\right)$ and second$\operatorname{crossover}\left(\sigma=0, \sigma_{f}=-0.1, F=.002\right)$, and 2:1 resonant non-crossover $\left(\sigma=\sigma_{f}=0, F=.002\right)$ cables are comparatively visualized in Figs.9a, 9b and 9c, respectively. The solid (dashed) lines denote time-varying spatial distributions over a half period $(t=0-.5 \tau)$ of forced vibrations with NC (CC) model. The hidden vertical axes in Figs.9a, 9b and 9c have a range of [-.005,.005], $[-.0021, .0021]$ and $[-.001, .002]$, respectively. Overall, it is evident that, due to mixed modal interactions at first (Fig.9a) and second (Fig.9b) crossovers, the 1:1 resonant displacements are actually asymmetric with respect to middle span, whereas the 2:1 resonant ones, due to purely symmetric modal interaction, still exhibit the symmetric spatial character (Fig.9c). These results are in qualitative agreement with numerical and analytical results in [4] and [6], which highlighted 1:1 and 2:1 resonant modes in non-linear free vibrations, respectively.

Since the resonant $\left(a_{r}-a_{s}\right)$ amplitudes of $\mathrm{NC} / \mathrm{CC}$ models are quantitatively different (see Figs.3, 5 and 7), there are quantitative discrepancies in the relevant displacement distributions of all cables. For 1:1 resonant cables, the qualitative agreement in Figs.9a and $9 \mathrm{~b}$ is satisfactory, whereas there are some qualitative differences in 2:1 resonant profiles. Namely, as mostly visible at $t=.4 \tau$ or $.5 \tau$ in Fig.9c, the NC model shows the symmetric second mode-like configuration (see Fig. 2) because the driven $a_{r}$ amplitude $(\approx .000784)$ dominates the coupled response and contributions from the corresponding $a_{s}$ amplitude $(\approx .000168)$ are relatively small (Fig.7a), whereas the $\mathrm{CC}$ model exhibits a clear combination of $2^{\text {nd }}$ and $5^{\text {th }}$ modal shapes because the amplitudes $\left(a_{r} \approx .000547, a_{s} \approx .000415\right)$ of the two participating modes are nearly comparable to each other (Fig.7b). Thus, the largest difference at $t=.4 \tau$ or $.5 \tau$ is seen in Fig.9c at cable midspan, where the maximum static curvature and quadratic drift effects take place.

With reference to Figs.3a and $3 \mathrm{~b}$ of the 1:1 resonant first-crossover cable, the spatio-temporal dynamic tensions of NC model (solid lines) are shown in Figs.10a and 10c for $\sigma_{f}=0$ and $\sigma_{f} \approx$ -.12 , respectively, in comparison with the corresponding spatially-constant tensions of CC model 
(dashed lines) in Figs.10b and 10d. For both $\sigma_{f}$, dynamic tensions of the associated stable higheror lower-amplitude uncoupled $a_{s}$ responses (dotted lines) are also evaluated, to better determine actual maximum stresses. In all cases, the $T_{d}$ values - which can be either positive (tensile) or negative (compressive) - are normalized with respect to the associated maximum static tension $T_{H}$, and evaluated over a half period $\tau$, whose time steps sequence follows the arrow in Fig.10.

Overall, the spatially asymmetric (symmetric) character of the coupled (uncoupled) 1:1 resonant configurations of $\mathrm{NC}$ model, (see, e.g., Fig.9a for the coupled configuration), reflects the asymmetric (symmetric) feature of dynamic tension distributions in Figs.10a and 10c. With $\sigma_{f}=0$, some NC tension distributions of coupled and uncoupled responses (Fig.10a) are nearly spatially uniform and trivial, e.g. at $t=0.2 \tau$, because the cable vibrates in a neighborhood of its static equilibrium, giving rise to small displacement gradients (see, e.g., Fig.9a). In both Figs.10a and $10 \mathrm{~b}$, some of the estimated dynamic tensions of the uncoupled responses seem to be more tensile $(t=0-.2 \tau)$ or compressive $(t=0.4 \tau-.5 \tau)$ than those of the coexisting coupled responses. Thus, the former produces larger non-linear tensile or compressive stresses than the latter. With NC (CC) model, the maximum tensile stress of uncoupled responses occurs at $t=0$, at node 37 , with $T_{d} / T_{H} \approx .3709$ (.3202), whereas that of coupled responses occurs at $t=0$, at node 28 , with $T_{d} / T_{H} \approx .3492$ (.2208). Apart from overlooking the essential asymmetric spatial features, the CC model predicts lower dynamic stresses in both coupled/uncoupled responses. Yet, the greater difference ( $36.77 \%$ ) with respect to NC model actually occurs in the coupled response.

With $\sigma_{f} \approx-.12$, because of low-amplitude responses, it is obvious that all of the dynamic tension distributions of uncoupled NC solution (Fig.10c) are nearly spatially uniform, like those of CC model (Fig.10d); consequently both models have comparable values at each time step and one may use the $\mathrm{CC}$ model for such situation. On the other hand, there are greater differences in the larger-amplitude coupled responses, where the associated spatial variation of NC dynamic strain is actually important putting into evidence a strong asymmetric feature. The coupled 
responses also produce larger dynamic stresses than the uncoupled ones, with respect to the case $\sigma_{f}=0$. With NC model, the maximum tensile (compressive) stress at $t=0(t=-.5 \tau)$ of coupled response occurs at node $12(45)$ with $T_{d} / T_{H} \approx .6791$ (-.5269), whereas that of uncoupled response occurs at node 38 (26) with $T_{d} / T_{H} \approx .1569$ (-.1546). With CC model, the maximum tensile (compressive) stresses of coupled/uncoupled responses occur at $t=0(t=.5 \tau)$ with $T_{d} / T_{H} \approx .4004$ (-.3025) and .1564 (-.1526), respectively. Consequently, there are greater quantitative differences $(>40 \%)$ in dynamic stresses of coupled responses provided by the two models, with the NC one furnishing a larger time excursion range.

Depending on cable kinematic modeling, it is worth remarking that not all of the obtained stable large-amplitude results always guarantee positive cable total tension during vibration. We have just drawn overall pictures and made a thorough comparison of non-linear responses with different modeling. In spite of this, analyses based on NC model have highlighted how dynamic tensions induced in even shallow cables significantly change in both space and time. This accounted variability, on the one hand, allows us to accurately predict the stress demand for cables; on the other hand, it allows us to cope with the associated finite-amplitude vibrations, whether unavoidable, by properly improving other cable properties such as damping [20, 21]. In contrast, analyses based on CC model, besides being definitely constrained to spatially-constant tensions, give rise to considerably underestimated dynamic stresses. This may result in unreliable design, particularly when compressive forces might actually occur.

\section{CONCLUSIONS}

Non-linear forced vibrations of shallow horizontal cables due to simultaneous primary external and planar 1:1 or 2:1 internal resonances are analytically investigated with the kinematically non-condensed vs condensed modeling. The former accounts for spatio-temporal dynamic tension modification and explicitly captures non-linear coupling of longitudinal/vertical displacements, whereas, in the latter, meaningful higher-order effects of longitudinal dynamic 
deformation are eliminated, thereby entailing spatially-constant dynamic tension. Second-order quadratic non-linear modal contributions and orthogonality properties of symmetric/antisymmetric modes are discussed, clarifying the modal participating capacity between 1:1/2:1 (mixed/symmetric) resonant cables (modal interactions), and between crossover/non-crossover cables. Analyses put into evidence the significance of accounting for both resonant/non-resonant modes in all resonance cases, and meaningful differences in the values and/or sign of effective non-linear coefficients with the two distinctive models.

Coupled/uncoupled, steady-state/periodic responses and their stability (bifurcations) are evaluated by means of frequency- and force-response diagrams. Direct numerical integrations are utilized to confirm the existence of steady-state or modulated amplitude responses. Moreover, coupled displacement profiles accounting for spatial corrections from all of the retained modes due to quadratic nonlinearities are constructed, and space-time varying non-linear dynamic tensions are analyzed. Results shed light on the actual spatially asymmetric/symmetric character of both dynamic configurations and tension distributions furnished by the non-condensed model.

Depending on internal resonance condition and system elasto-geometric/control parameters, the parametric investigation highlights that, even for shallow cables, the condensed model may lead to significant quantitative and/or qualitative discrepancies in non-linear dynamic responses, bifurcation properties, as well as non-linear tensile/compressive stresses, with respect to the noncondensed model. Actual errors are seen to be significant in coupled - particularly higheramplitude - responses, with respect to the uncoupled ones, because the dynamics of the former are thoroughly controlled by the differences in all of the interaction coefficients, whereas those of the latter are solely controlled by the difference in one of them.

For this reason, to obtain more accurate approximate solutions and overcome some inherent drawbacks of the condensed model, it is recommended, based on our explorations, to consider the more general non-condensed model in the analytical-numerical treatment of cable non-linear resonant dynamics. In this respect, the drawbacks are expected to be somehow enhanced 
whenever considering a multiple internal resonance involving coupled planar/non-planar modes, as well as highly extensible and/or non-shallow (i.e., non-parabolic) suspended cables.

Acknowledgement: Dr. Narakorn Srinil is extremely grateful to the support from the University of Rome 'La Sapienza', Italy, through a Postdoctoral Fellowship.

\section{References}

1. Rega G., Nonlinear dynamics of suspended cables, Part I. Modeling and analysis, Part II. Deterministic phenomena, ASME Appl. Mech. Rev. 57, 2004, 443-514.

2. Irvine H.M., Caughey T.K., The linear theory of free vibrations of a suspended cable, Proceeding of the Royal Society of London Series A 341, 1974, 229-315.

3. Srinil N., Rega G., Chucheepsakul S., Large amplitude three-dimensional free vibrations of inclined sagged elastic cables, Nonlinear Dyn. 33, 2003, 129-154.

4. Srinil N., Rega G., Chucheepsakul S., Three-dimensional nonlinear coupling and dynamic tension in the large amplitude free vibrations of arbitrarily sagged cables, J. Sound Vib. 269, 2004, 823-852.

5. Srinil N., Rega G., Chucheepsakul S., Two-to-one resonant multi-modal dynamics of horizontal/inclined cables, Part I. Theoretical formulation and model validation, Nonlinear Dyn., 2007, DOI: $10.1007 / \mathrm{s} 11071-006-9086-0$.

6. Srinil N., Rega G., Two-to-one resonant multi-modal dynamics of horizontal/inclined cables, Part II. Internal resonance activation, reduced-order models and nonlinear normal modes, Nonlinear Dyn., 2007, DOI: $10.1007 / \mathrm{s} 11071-006-9087-z$.

7. Lacarbonara W., Rega G., Nayfeh A.H., Resonant nonlinear normal modes, Part I. Analytical treatment for structural one-dimensional systems, Int. J. Non-linear Mech. 38, 2003, 851-872.

8. Lacarbonara W., Rega G., Resonant nonlinear normal modes, Part II. Activation/orthogonality conditions for shallow structural systems, Int. J. Non-linear Mech. 38, 2003, 873-887.

9. Rao G.V., Iyengar R.N., Internal resonance and non-linear response of a cable under periodic excitation, J. Sound Vib. 149, 1991, 25-41.

10. Perkins N.C., Modal interactions in the non-linear response of elastic cables under parametric/external excitation, Int. J. Non-linear Mech. 27, 1992, 233-250.

11. Lee C.L., Perkins N.C., Nonlinear oscillations of suspended cables containing a two-to-one internal resonance, Nonlinear Dyn. 3, 1992, 465-490.

12. Pakdemirli M., Nayfeh S.A., Nayfeh A.H., Analysis of one-to-one autoparametric resonance in cables-discretization vs. direct treatment, Nonlinear Dyn. 8, 1995, 65-83.

13. Zhao Y.Y., Wang L.H., Chen D.L., Jiang L.Z., Non-linear dynamic analysis of the twodimensional simplified model of an elastic cable, J. Sound Vib. 255, 2002, 43-59. 
14. Rega G., Lacarbonara W., Nayfeh A.H., Chin C.M., Multiple resonances in suspended cables: Direct versus reduced-order models, Int. J. Non-linear Mech. 34, 1999, 901-924.

15. Nayfeh A.H., Arafat H.N., Chin C.M., Lacarbonara W., Multimode interactions in suspended cables, J. Vib. Control 8, 2002, 337-387.

16. Zheng G., Ko G.M., Ni Y.Q., Super-harmonic and internal resonances of a suspended cable with nearly commensurable natural frequencies, Nonlinear Dyn. 30, 2002, 55-70.

17. Gattulli V., Martinelli L., Perotti F., Vestroni F., Nonlinear oscillations of cables under harmonic loading using analytical and finite element models, Comput. Methods Appl. Mech. Eng. 193, 2004, 69-85.

18. Srinil N., Rega G., Resonant non-linear dynamic responses of horizontal cables via kinematically non-condensed/condensed modeling, The $3^{\text {rd }}$ European Conference on Computational Mech. Solids, Struct. Coupled Problems in Eng., Lisbon, 2006, 1-20.

19. Seydel R., Practical Bifurcation and Stability Analysis, Springer, New York, 1994.

20. Gaul, L., The influence of damping on waves and vibrations, Mechanical Systems and Signal Processing 13, 1999, 1-30.

21. Kounadis A.N., Hamiltonian weakly damped autonomous systems exhibiting periodic attractors, ZAMP 57, 2006, 324-350. 


\section{List of Figures}

\section{Figure}

1 The schematic model of a shallow horizontal cable.

2 Planar frequency spectrum and crossover phenomena of horizontal cables, with vertical shapes of $r$ and $s$ modes involved in internal resonances.

3 A comparison of frequency-response curves and bifurcations of 1:1 resonant first-crossover cable $(\lambda / \pi \approx 2.02)$ with $\sigma=0, F=.002$ : (a) NC and (b) CC model.

4 A comparison of force-response diagrams and bifurcations of 1:1 resonant first-crossover cable $(\lambda / \pi \approx 2.02)$ with $\sigma=0$, and $\sigma_{f}=-0.12(\mathrm{a}, \mathrm{b})$ or $\sigma_{f}=0.12(\mathrm{c}, \mathrm{d}):(\mathrm{a}, \mathrm{c}) \mathrm{NC}$ and (b, d) CC model.

5 A comparison of frequency-response curves and bifurcations of 1:1 resonant second-crossover cable $(\lambda / \pi \approx 4.03$ ) with $\sigma=0, F=.002$ : (a) $\mathrm{NC}$ and (b) $\mathrm{CC}$ model, with corresponding dynamic solution branches (c) and (d) between the two Hopf bifurcations in (a) and (b), respectively.

6 Amplitude time histories from direct numerical integrations of 1:1 resonant second-crossover cable $(\lambda / \pi \approx 4.03)$ with $\sigma=0$ and $(\mathrm{a}, \mathrm{b}) \sigma_{f}=-0.1, F=.002$, (c, d) $\sigma_{f}=0, F=.002,(\mathrm{e}, \mathrm{f}) \sigma_{f}=0, F$ $=.005:(\mathrm{a}, \mathrm{c}, \mathrm{e}) \mathrm{NC}$ and $(\mathrm{b}, \mathrm{d}, \mathrm{f}) \mathrm{CC}$ model: solid (dashed) lines denote $a_{r}\left(a_{s}\right)$.

7 A comparison of frequency-response curves and bifurcations of 2:1 resonant non-crossover cable $(\lambda / \pi \approx 2.95$ ) with $\sigma=0, F=.002$ : (a) NC and (b) CC model.

8 A comparison of force-response diagrams and bifurcations of 2:1 resonant non-crossover cable $\left(\lambda / \pi \approx 2.95\right.$ ) with $\sigma=0, \sigma_{f}=0$ : (a) NC and (b) CC model.

9 A comparison of non-linear coupled dynamic configurations over a half period of forced vibration with $\mathrm{NC}$ (solid lines) and $\mathrm{CC}$ (dashed lines) models: (a) $\lambda / \pi \approx 2.02, \sigma=0, \sigma_{f}=0$, (b) $\lambda / \pi \approx 4.03, \sigma=0, \sigma_{f}=-.1$, (c) $\lambda / \pi \approx 2.95, \sigma=0, \sigma_{f}=0$.

10 A comparison of non-linear dynamic tension distributions associated with coupled (solid and dashed lines) and uncoupled (dotted lines) responses over a half period of forced vibration of 1:1 resonant first-crossover cable $(\lambda / \pi \approx 2.02)$ with $(\mathrm{a}, \mathrm{b}) \sigma_{f}=0$ or $(\mathrm{c}, \mathrm{d}) \sigma_{f} \approx-0.12$ : $(\mathrm{a}, \mathrm{c}) \mathrm{NC}$ and (b, d) CC model.

\section{List of Tables}

\section{Table}

1 Given parameters and properties for 1:1 and 2:1 resonant NC cables.

2 A comparison of second-order quadratic modal contributions of 1:1 resonant first-crossover $(\lambda / \pi \approx 2.02)$ and $2: 1$ resonant non-crossover $(\lambda / \pi \approx 2.95) \mathrm{NC}$ cables.

3 A comparison of second-order quadratic modal contributions of second-crossover NC cable $(\lambda / \pi \approx 4.03)$ exhibiting $1: 1$ or $2: 1$ internal resonance.

4 A comparison of $\mathrm{NC}$ and $\mathrm{CC}$ effective non-linear coefficients: (a) 1:1 resonant first-crossover $(\lambda / \pi \approx 2.02)$ and $2: 1$ resonant non-crossover $(\lambda / \pi \approx 2.95)$ cables; (b) $1: 1$ or $2: 1$ resonant second$\operatorname{crossover}(\lambda / \pi \approx 4.03)$ cable. 


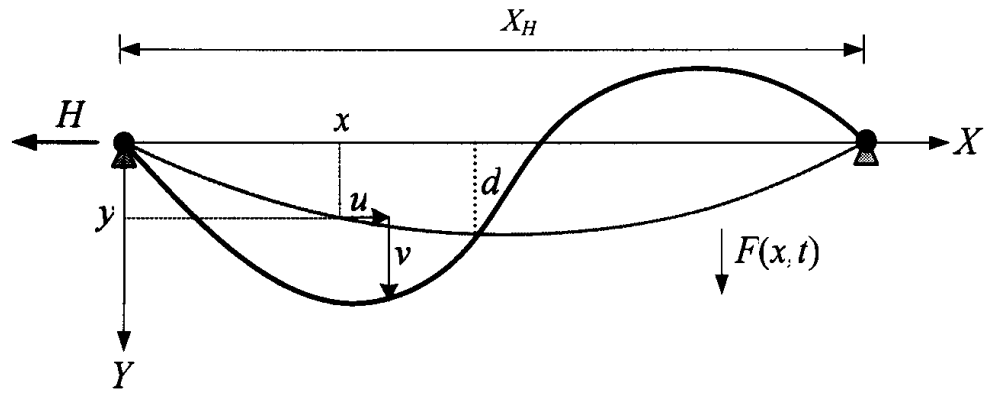

Figure 1

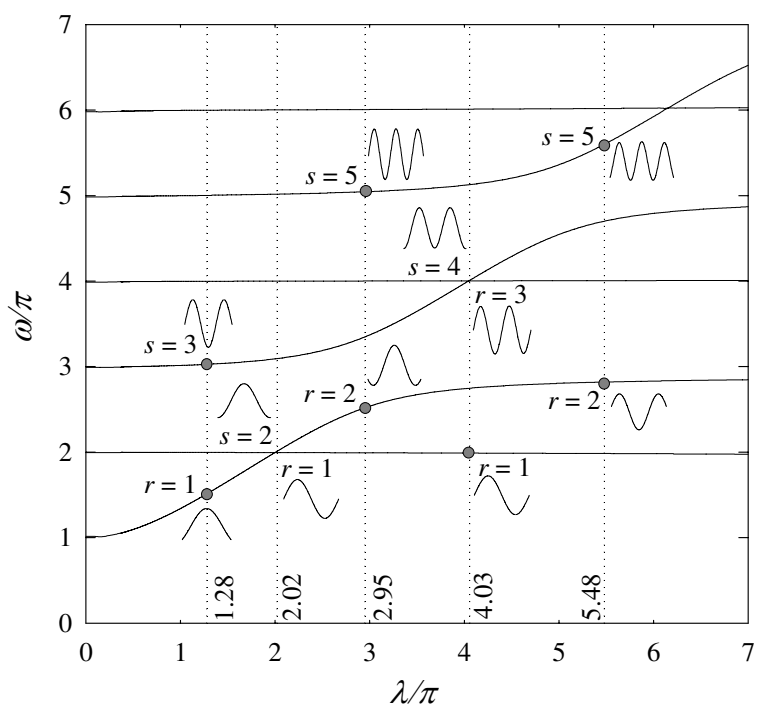

$\underline{\text { Figure } 2}$ 

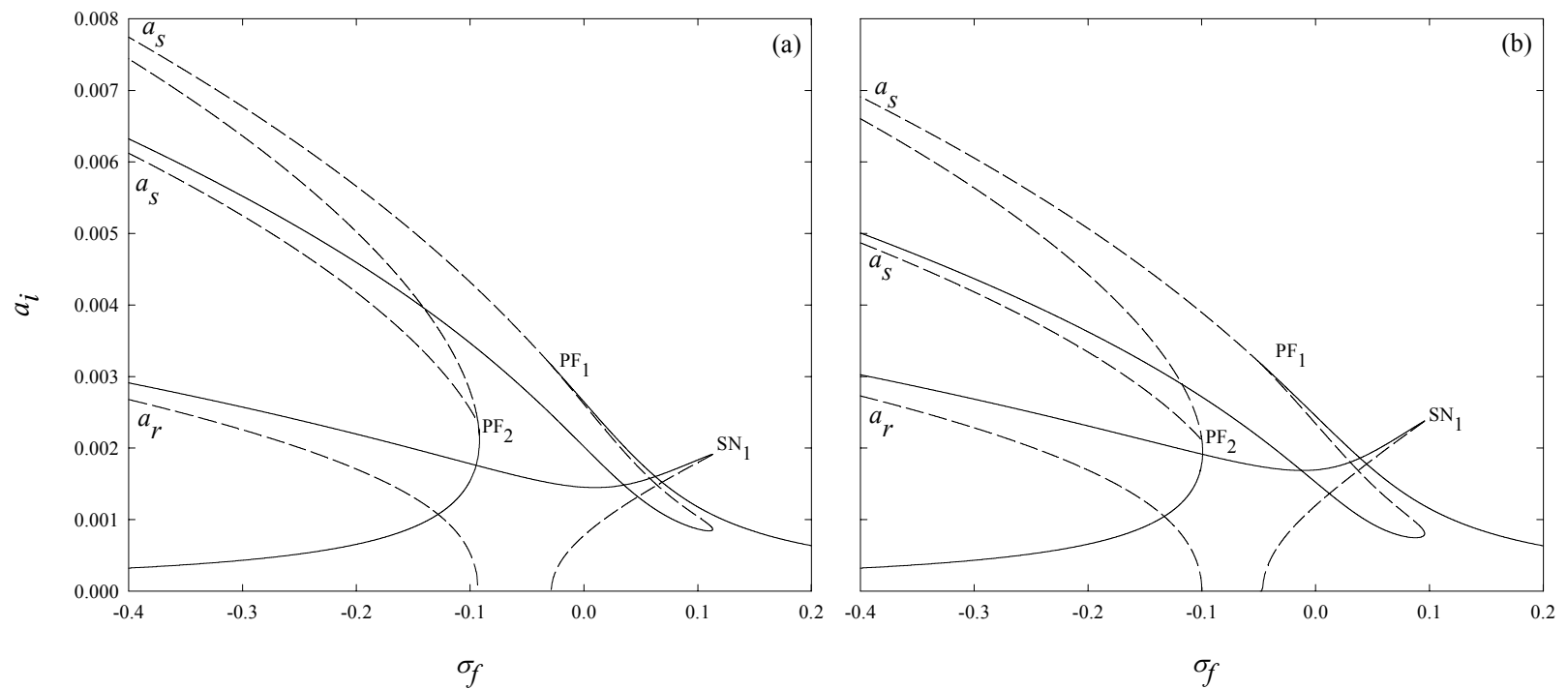

$\underline{\text { Figure } 3}$ 

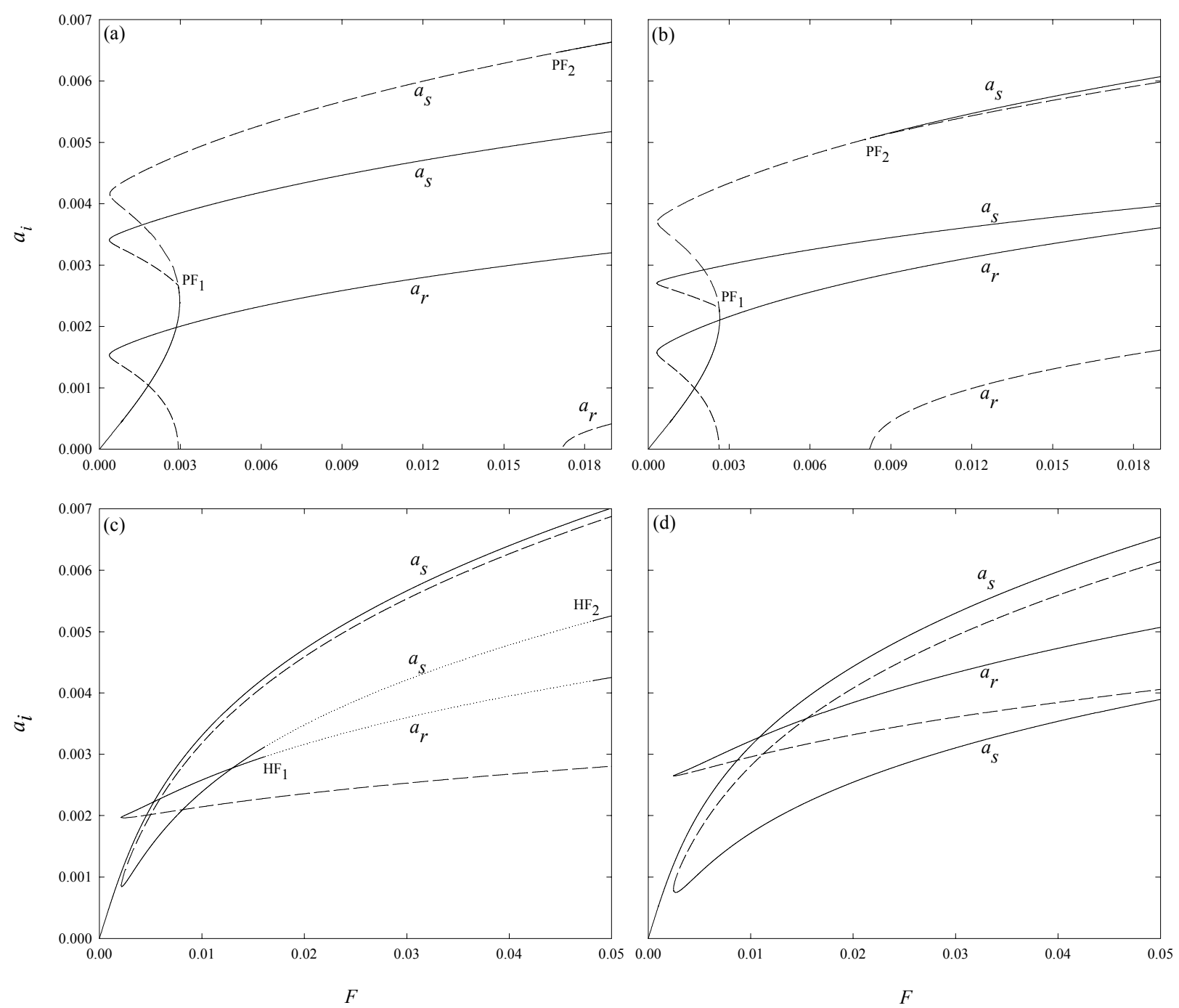

Figure 4 

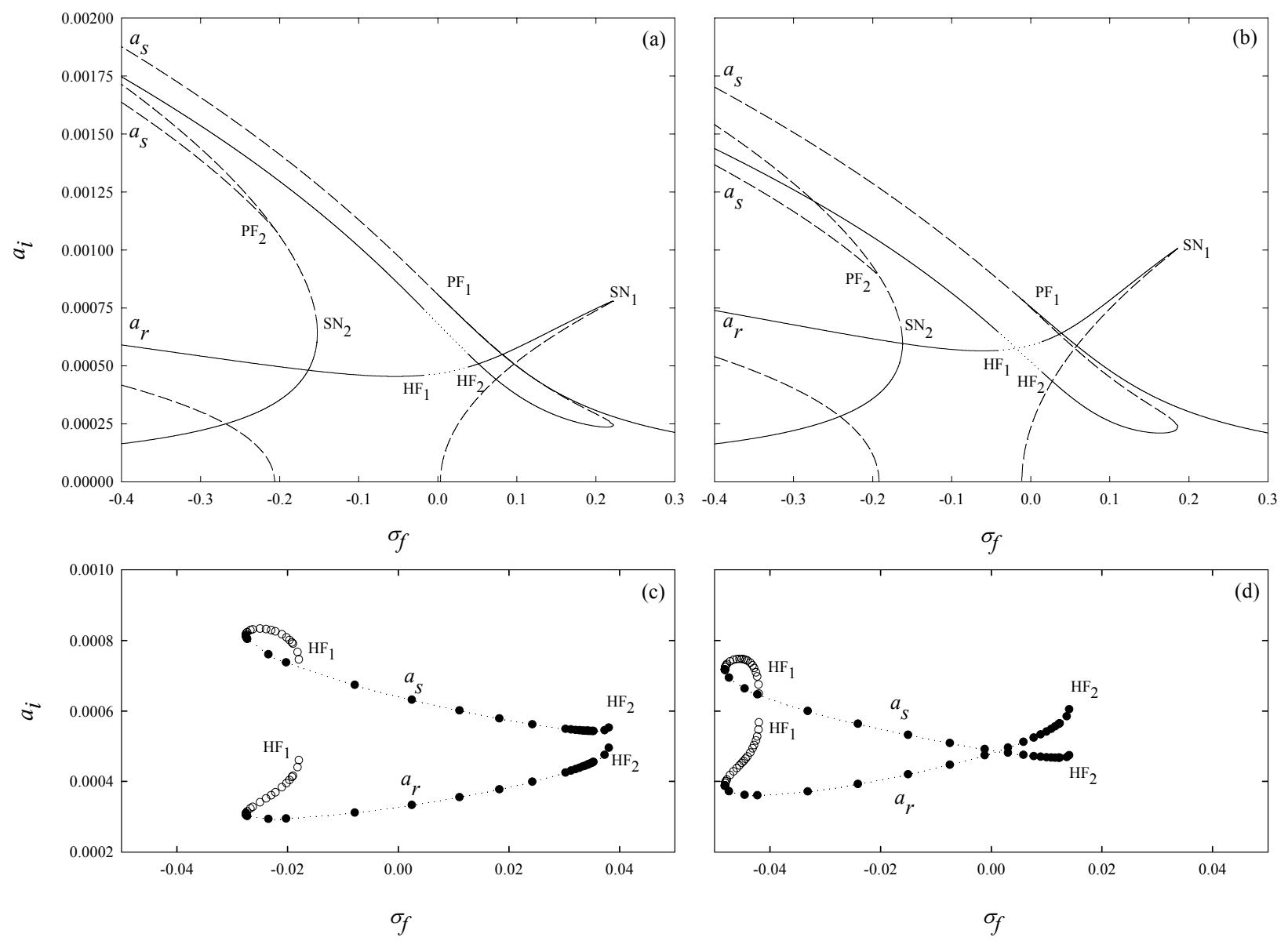

Figure 5 

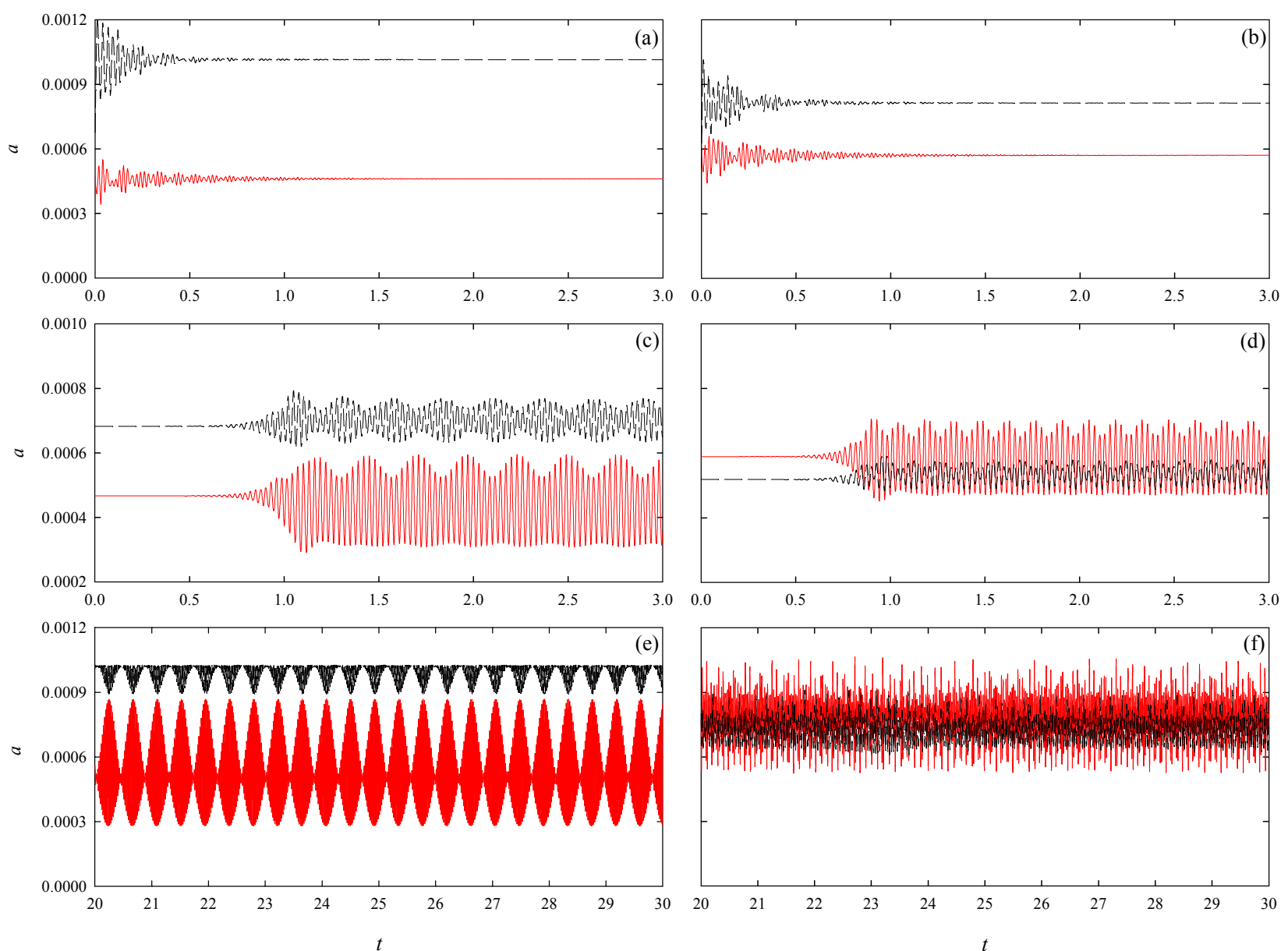

$\underline{\text { Figure } 6}$ 

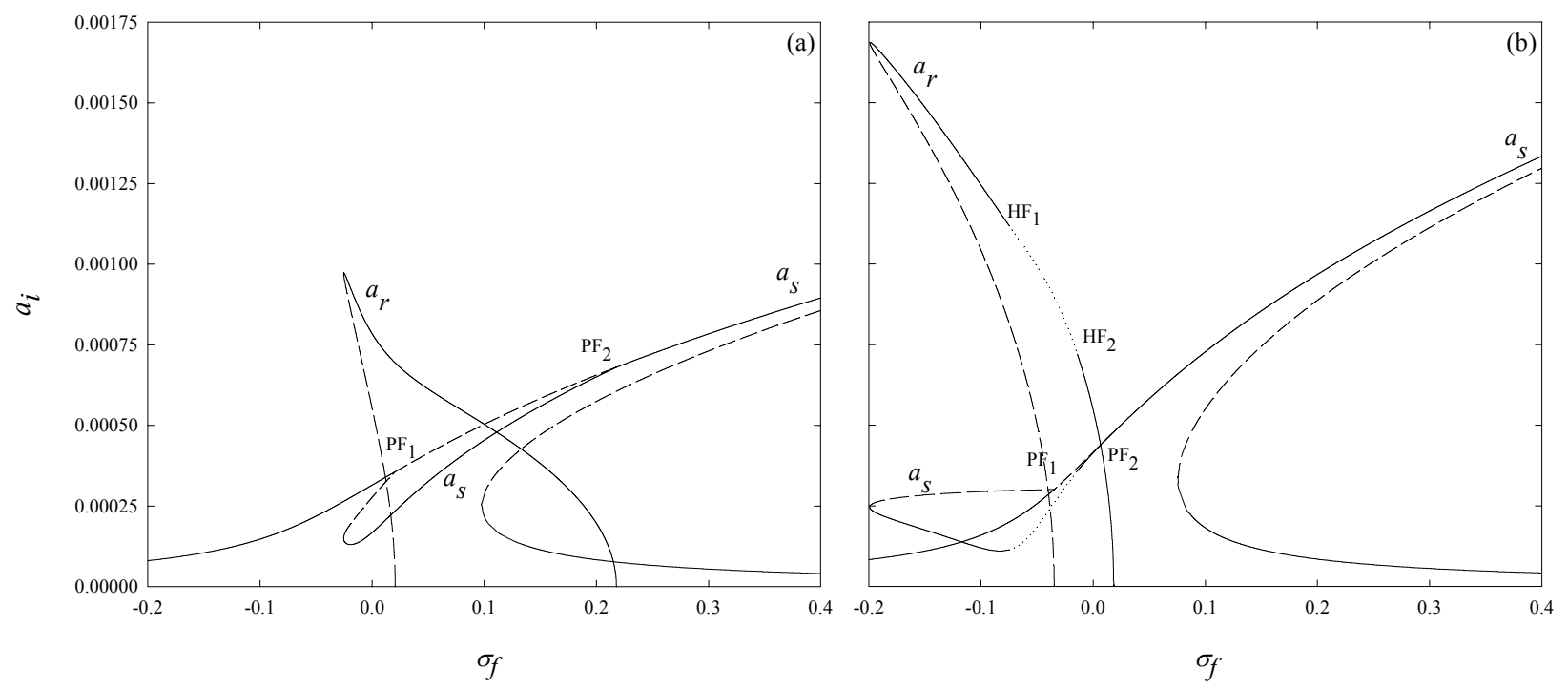

Figure 7
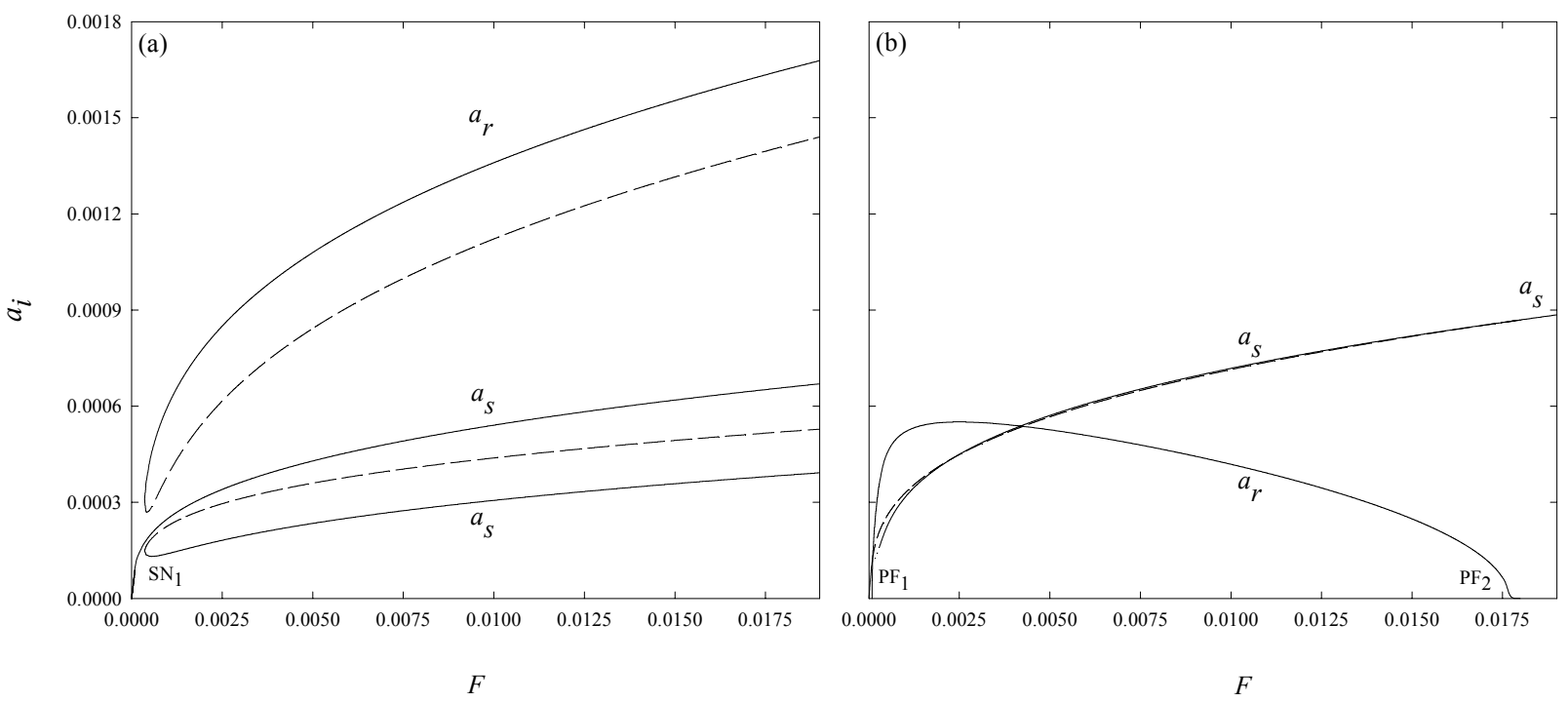

Figure 8 
(a)
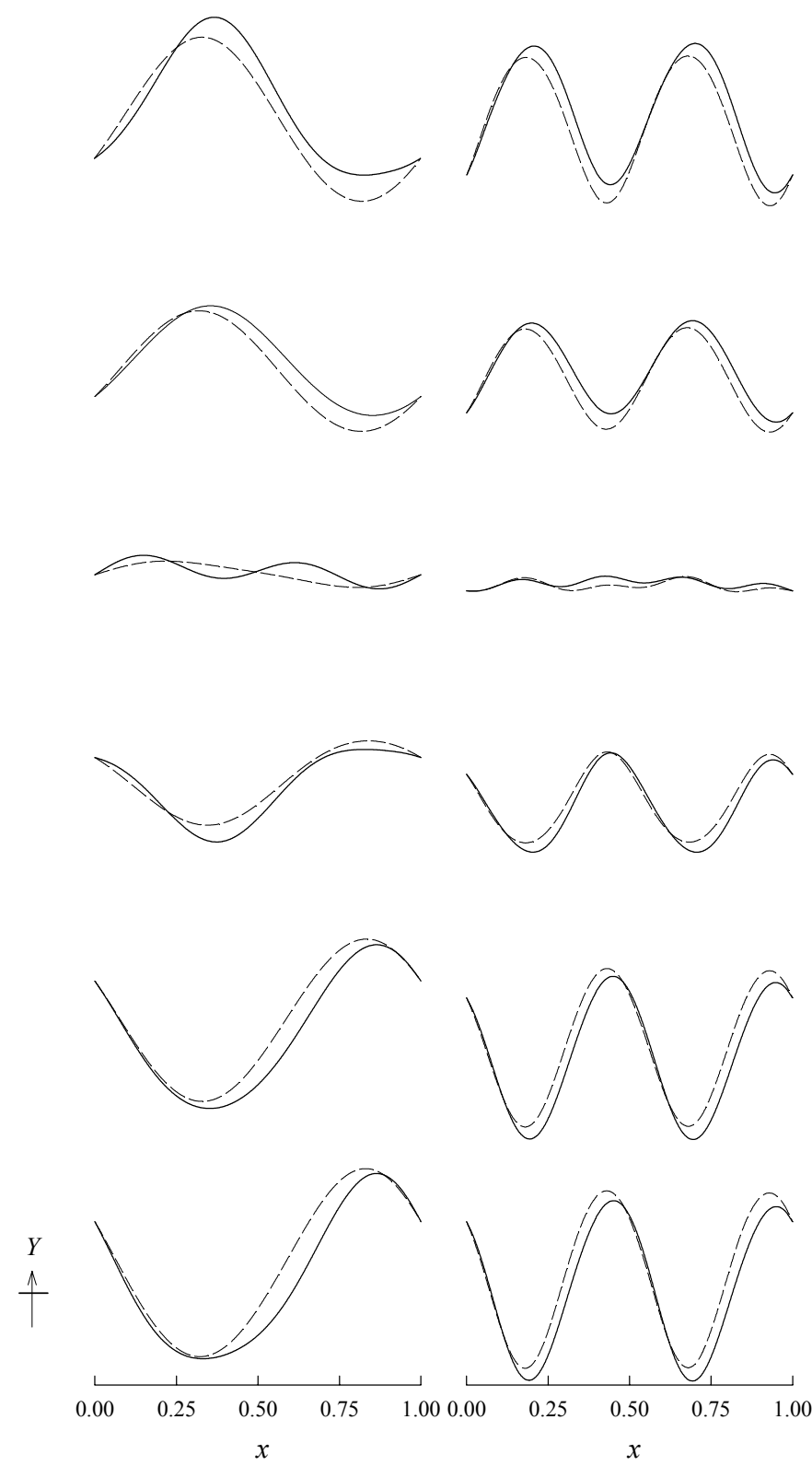

(c)
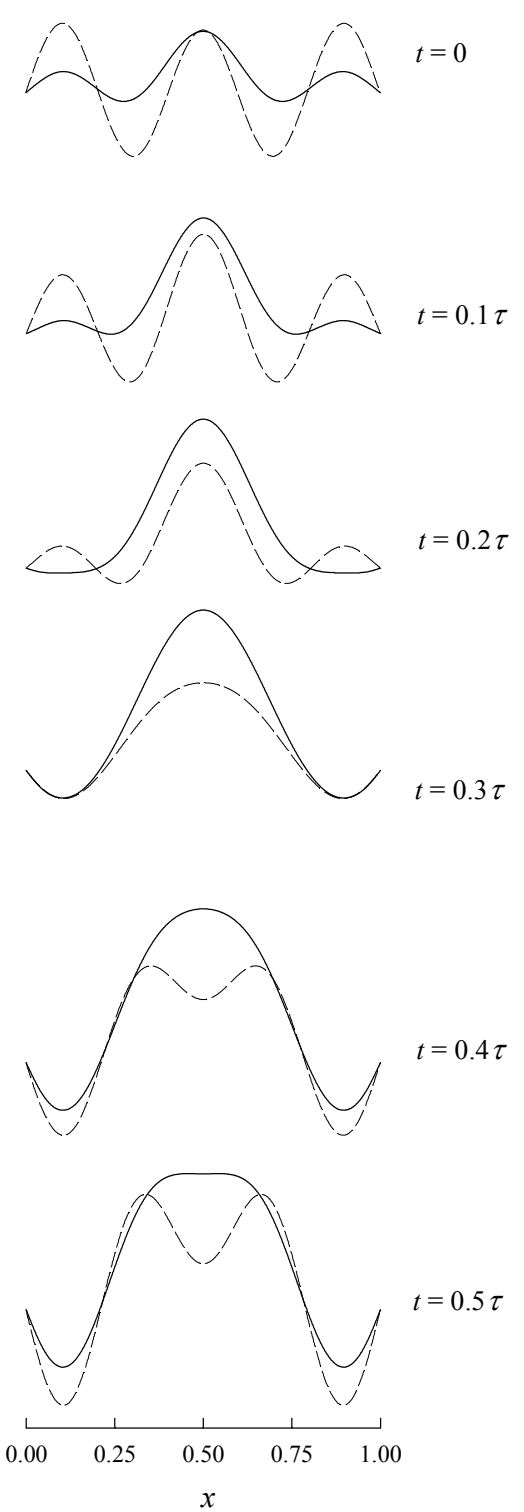

Figure 9 

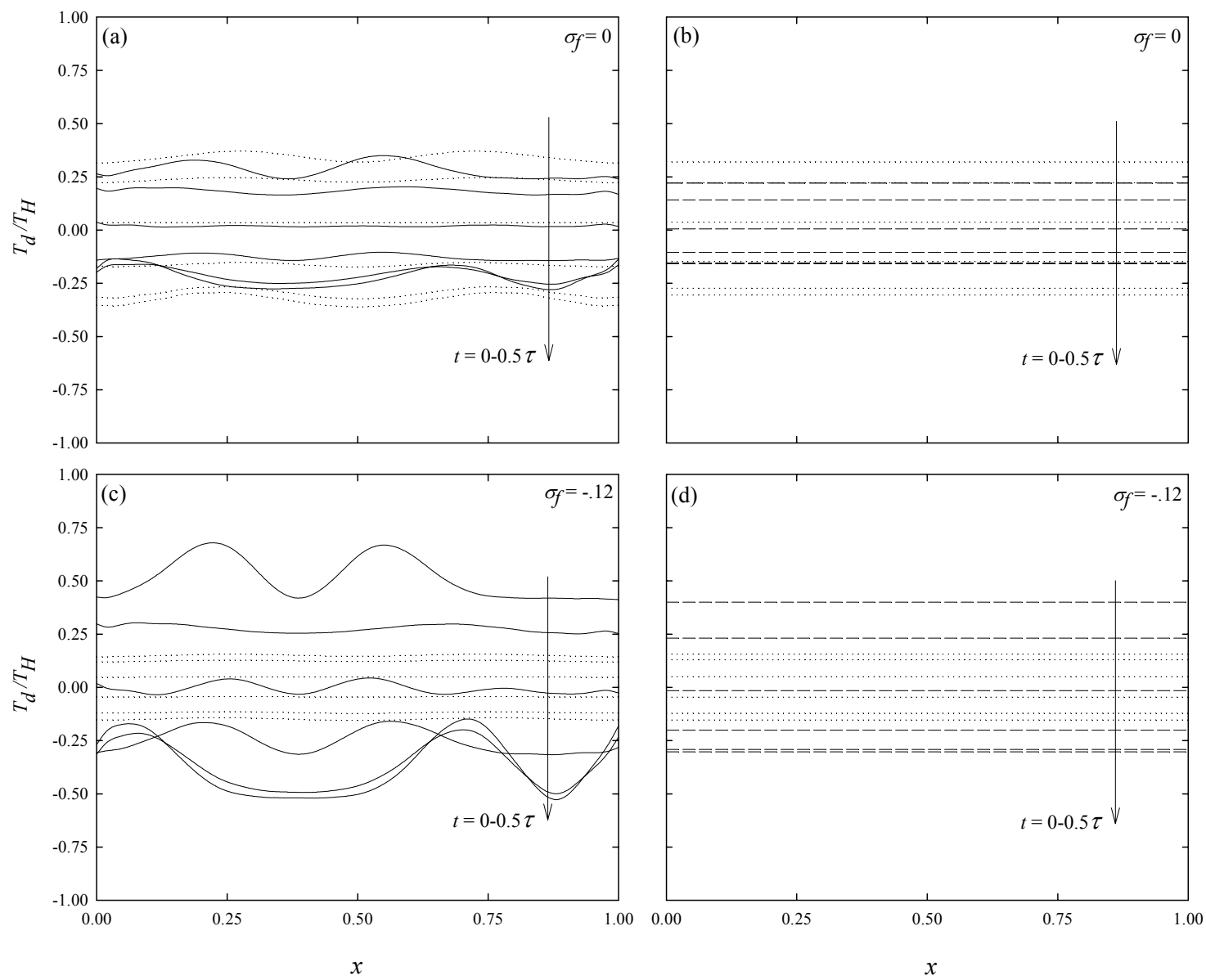

Figure 10 
Table 1

\begin{tabular}{|c|c|c|c|c|c|c|c|c|c|}
\hline \multirow{2}{*}{$\lambda / \pi$} & \multirow{2}{*}{$\alpha$} & \multirow{2}{*}{$d$} & \multirow{2}{*}{$r: s$} & \multicolumn{2}{|c|}{$r-s$} & \multirow{2}{*}{$N$} & \multirow{2}{*}{$\omega_{r}$} & \multirow{2}{*}{$\omega_{s}$} & \multirow{2}{*}{$\Upsilon_{s}=\int_{0} \varphi_{s} d x$} \\
\hline & & & & Order & Mode* & & & & \\
\hline 2.02 & 642.72 & .031 & $1: 1$ & $1-2$ & A-S & 20 & 6.259 & 6.305 & .8155 \\
\hline 2.95 & 828.18 & .040 & $1: 2$ & $2-5$ & S-S & 20 & 7.914 & 15.824 & .2604 \\
\hline 4.03 & 1018.26 & .049 & $\{1: 1$ & $3-4$ & A-S & 40 & 12.537 & 12.544 & .8187 \\
\hline & & & $1: 2$ & $1-4$ & A-S & 40 & 6.223 & 12.544 & .8187 \\
\hline
\end{tabular}

Table 2

\begin{tabular}{|c|c|c|c|c|c|c|c|}
\hline \multirow{3}{*}{$m^{*}$} & \multicolumn{7}{|c|}{ Modal contributions (\%) } \\
\hline & \multicolumn{4}{|c|}{$1: 1$} & \multicolumn{3}{|c|}{$2: 1$} \\
\hline & $K_{r r}^{q}$ & $K_{s s}^{q}$ & $K_{r s}^{q}$ & $K_{3}^{q}$ & $K_{r r}^{q}$ & $K_{s s}^{q}$ & $K_{r s}^{q}$ \\
\hline $1^{\mathrm{A}}$ & $\underline{0}$ & $\underline{0}$ & 44.344 & 109.303 & 0 & 0 & 0 \\
\hline $2^{S}$ & $92.96 \overline{6}$ & $92.44 \overline{9}$ & 66.414 & -18.639 & 97.266 & 43.370 & $\underline{83.415}$ \\
\hline $3^{S}$ & 3.554 & 4.237 & -8.948 & 11.152 & 2.473 & 37.419 & -5.531 \\
\hline $4^{\mathrm{A}}$ & 0 & 0 & -0.903 & 0.002 & 0 & 0 & 0 \\
\hline $5^{S}$ & 2.876 & 2.742 & -0.704 & -1.579 & $\underline{0.194}$ & 19.141 & 22.493 \\
\hline $6^{\mathrm{A}}$ & 0 & 0 & 0.001 & 0.001 & $\overline{0}$ & 0 & 0 \\
\hline $7^{S}$ & 0.459 & 0.435 & -0.153 & -0.187 & 0.054 & 0.178 & -0.421 \\
\hline$:$ & : & : & : & : & : & $:$ & : \\
\hline $10^{\mathrm{A}}$ & $\dot{0}$ & $\dot{0}$ & $\dot{0}$ & $\dot{0}$ & $\dot{0}$ & 0 & 0 \\
\hline
\end{tabular}

Table 3

\begin{tabular}{|c|c|c|c|c|c|c|c|}
\hline \multirow{3}{*}{$m^{*}$} & \multicolumn{7}{|c|}{ Modal contributions (\%) } \\
\hline & \multicolumn{4}{|c|}{$1: 1$} & \multicolumn{3}{|c|}{$2: 1$} \\
\hline & $K_{r r}^{q}$ & $K_{s s}^{q}$ & $K_{r s}^{q}$ & $K_{3}^{q}$ & $K_{r r}^{q}$ & $K_{s s}^{q}$ & $K_{r s}^{q}$ \\
\hline $1^{\mathrm{A}}$ & 0 & 0 & 0.030 & 0.044 & $\underline{0}$ & $\underline{0}$ & $\underline{17.583}$ \\
\hline $2^{S}$ & 17.404 & 18.396 & -17.178 & 1.565 & $7.17 \overline{6}$ & 18.396 & -30.872 \\
\hline $3^{\mathrm{A}}$ & $\underline{0}$ & $\underline{0}$ & $\underline{53.168}$ & 108.791 & 0 & 0 & 0.031 \\
\hline $4^{\mathrm{S}}$ & $\underline{73.60 \overline{8}}$ & $71.14 \overline{4}$ & $\overline{77.815}$ & -17.726 & 69.407 & 71.144 & $\underline{138.696}$ \\
\hline $5^{\mathrm{S}}$ & $\overline{8.180}$ & 9.754 & $-\overline{12.260}$ & $\overline{5.816}$ & $\overline{20.982}$ & 9.754 & $\overline{-22.541}$ \\
\hline $6^{\mathrm{A}}$ & 0 & 0 & -0.005 & 0.025 & 0 & 0 & 0.269 \\
\hline $7^{\mathrm{S}}$ & -0.965 & -0.945 & -1.206 & 2.821 & 1.734 & -0.945 & -2.213 \\
\hline $8^{\mathrm{A}}$ & 0 & 0 & 0.185 & 0.001 & 0 & 0 & 0.009 \\
\hline $9^{\mathrm{S}}$ & 1.288 & 1.197 & -0.334 & -1.060 & 0.428 & 1.197 & -0.589 \\
\hline$:$ & & : & : & : & : & : & : \\
\hline $15^{\mathrm{S}}$ & 0.062 & 0.058 & -0.032 & -0.030 & 0.039 & 0.058 & -0.056 \\
\hline
\end{tabular}


Table 4

(a)

\begin{tabular}{crrrr}
\hline \multirow{2}{*}{$1: K$} & \multicolumn{1}{c}{$\mathrm{N}$} & \multicolumn{1}{c}{$2: 1$} \\
\cline { 2 - 5 } & \multicolumn{1}{c}{$\mathrm{NC}$} & - & $\mathrm{NC}$ & \multicolumn{1}{c}{$\mathrm{CC}$} \\
\hline $\mathfrak{R}$ & - & - & 1207.261 & 1432.430 \\
$K_{r r}$ & -1623894.953 & -902564.584 & 937961.671 & 1924967.523 \\
$K_{s s}$ & 349674.770 & 441025.387 & -65898889.412 & -29278309.304 \\
$K_{r s}$ & 494314.116 & 683014.354 & -11398732.861 & 3289210.383 \\
$K_{3}$ & 351375.730 & 448725.018 & - & - \\
\hline
\end{tabular}

(b)

\begin{tabular}{crrrr}
\hline \multirow{2}{*}{,$K$} & \multicolumn{2}{c}{$1: 1$} & \multicolumn{2}{c}{$2: 1$} \\
\cline { 2 - 5 } & \multicolumn{1}{c}{$\mathrm{NC}$} & - & $\mathrm{CC}$ & \multicolumn{1}{c}{$\mathrm{CC}$} \\
\hline $\mathfrak{R}$ & - & -12350.868 & -12941.360 \\
$K_{r r}$ & -37034975.046 & -18777404.519 & -1787977.950 & -638996.879 \\
$K_{s s}$ & 12379545.241 & 15202808.717 & 12379545.241 & 15202808.717 \\
$K_{r s}$ & 8415149.890 & 13121299.228 & -3120155.675 & 979317.369 \\
$K_{3}$ & 8971440.226 & 11320816.901 & - & - \\
\hline
\end{tabular}

